\title{
Desafios de um Formalismo Hamiltoniano de Segunda Ordem
}

\author{
Lucas Perousa Bilar
}

\author{
DiSSERTAÇÃO APRESENTADA AO \\ INSTITUTO DE MATEMÁTICA E EsTATÍSTICA \\ DA UNIVERSIDAdE DE SÃo PAUlO \\ PARA OBTENÇÃO DO TÍTULO DE \\ MESTRE EM Cî̂NCIAS
}

Programa: Matemática Aplicada

Orientador: Prof. Frank Michael Forger

Durante o desenvolvimento deste trabalho o autor recebeu auxílio financeiro da CNPq

São Paulo

30 de Maio de 2021 



\title{
Desafios de um Formalismo Hamiltoniano de Segunda Ordem
}

\author{
Lucas Perousa Bilar
}

Esta é a versão original da dissertação elaborada pelo candidato Lucas Perousa Bilar, tal como submetida à Comissão Julgadora. 
Autorizo a reprodução e divulgação total ou parcial deste trabalho, por qualquer meio convencional ou eletrônico, para fins de estudo e pesquisa, desde que citada a fonte. 


\section{Agradecimentos}

Não teria chegado tão longe se não fosse pelo zelo e apoio de meus pais e pelo suporte oferecido pela minha futura esposa, Thais. Também gostaria de agradecer a todos os professores que enriqueceram a minha caminhada, em especial os professores e amigos Dr. Theo Allan Darn Zapata e Dr. Marco Cézar Barbosa Fernandes, grandes responsáveis pela minha base acadêmica em Física Matemática que foi imprescindível para a realização deste trabalho. 



\section{Resumo}

\section{Lucas Perousa Bilar. Desafios de um Formalismo Hamiltoniano de Segunda Ordem.}

Dissertação (Mestrado). Instituto de Matemática e Estatística, Universidade de São

Paulo, São Paulo, 2021.

A introdução de estruturas simpléticas generalizadas em fibrados de cojatos como âmbito matemático para o formalismo hamiltoniano de primeira ordem, tanto na mecânica clássica quanto na teoria clássica de campos, tem se mostrado muito útil para o entendimento do conceito físico de espaço de fase. É de se imaginar que essas mesmas estruturas possam desempenhar um papel semelhante num formalismo hamiltoniano de ordem superior. Entretanto, tal extensão a ordens superiores tem se mostrado extremamente desafiadora. O objetivo deste trabalho é detalhar alguns dos empecilhos já encontrados na passagem da primeira para a segunda ordem, evidenciando que tal extensão, se ela existir e se puder ser definida globalmente, deve ser altamente não-trivial.

Palavras-chave: Formalismo Hamiltoniano. Fibrados de Jatos. Fibrados de Cojatos. Estruturas Simpléticas Generalizadas. 



\section{Abstract}

\section{Lucas Perousa Bilar. Challenges of a Second Order Hamiltonian Formalism.}

Thesis (Master's). Institute of Mathematics and Statistics, University of São Paulo, São

Paulo, 2021.

The introduction of generalized symplectic structures in cojet bundles as the mathematical framework for the first order Hamiltonian formalism, in classical mechanics as well as in classical field theory, has proved to be very useful to understand the physical concept of phase space. It is reasonable to imagine that these same structures can play a similar role in a higher order Hamiltonian formalism. However, such an extension to higher orders has proved to be extremely challenging. The aim of this work is to spell out some of the obstacles already enocuntered in the transition from first to second order, showing that such an extension, if it exists at all and if it can be formulated globally, must be highly nontrivial.

Keywords: Hamiltonian Formalism. Jet Bundles. Cojet Bundles. Generalized Symplectic Structures. 



\section{Sumário}

$\begin{array}{ll}\text { Introduçăo } & 1\end{array}$

1 Fibrados de Jatos e Cojatos 5

1.1 Preliminares Algébricos . . . . . . . . . . . . . . . 5

1.2 Fibrados .............................. 6

1.3 Jatos e Cojatos de Primeira Ordem . . . . . . . . . . . . . . . . 7

1.4 Jatos e Cojatos de Segunda Ordem . . . . . . . . . . . . . . . . 9

2 Estruturas Simpléticas Generalizadas $\quad 15$

2.1 Estruturas Simpléticas . . . . . . . . . . . . . . . . . . . . . 15

2.2 Estruturas Multissimpléticas . . . . . . . . . . . . . . . 16

2.3 Estruturas Polissimpléticas . . . . . . . . . . . . . . . . 18

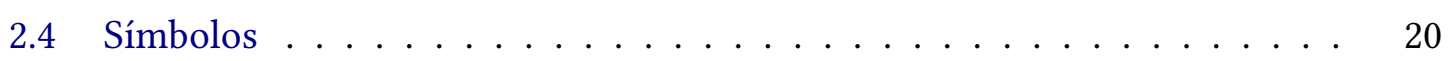

3 Formalismo Hamiltoniano de Primeira Ordem e Extensões 21

3.1 Estruturas Simpléticas e Multissimpléticas em Primeira Ordem . . . . . . 21

3.1 .1 Caso Unidimensional . . . . . . . . . . . . . . . . . . 21

3.1 .2 Caso Multidimensional . . . . . . . . . . . . . . . . . 23

3.2 Sobre a Extensão à Segunda Ordem . . . . . . . . . . . . . . . . . . . 24

3.2.1 Caso Unidimensional . . . . . . . . . . . . . . . . . . . . . 24

3.2 .2 Caso Multidimensional . . . . . . . . . . . . . . . 27

\section{Apêndices}

Anexos 



\section{Introdução}

Uma das consequências da evolução da Física durante o século 20 foi um protagonismo cada vez maior dos conceitos duais de estado e de observável na descrição do comportamento de sistemas físicos, na medida em que ficou cada vez mais claro que o tratamento tradicional provindo da mecânica newtoniana, em que o estado do sistema é descrito em termos das posições e das velocidades ou dos momentos das partículas que compõem este sistema, não era o único possível e precisava ser flexibilizado. De fato, tal flexibilização já ocorreu durante o desenvolvimento de áreas tais como termodinâmica, hidrodinâmica e mecânica estatística no século 19, onde os conceitos de estado e de observável sempre desempenham um papel fundamental, mas com uma interpretação às vezes bem diferente. Por exemplo, variáveis tais como temperatura ou pressão, em termos das quais se caracteriza o estado de um sistema na termodinâmica, "a priori" nada tem a ver com posições, velocidades ou momentos das partículas que compõem este sistema, e só "a posteriori", pela mecânica estatística, encontrou-se uma conexão entre as diferentes interpretações. Mas foi com o advento da mecânica quântica nos anos 20 do século 20 que se estabeleceu, de forma definitiva, uma visão mais ampla e mais abstrata dos conceitos de estado e de observável, uma vez que neste âmbito, mesmo referindo-se a apenas uma partícula, eles são representados, respectivamente, por vetores e por operadores em um espaço de Hilbert.

Em função desta nova visão, ganhou força a questão de como representar, de forma matematicamente adequada, o espaço de fase de um sistema, que é o conjunto de todos os seus estados possíveis. Felizmente, houve ao mesmo tempo uma forte evolução das ferramentas matemáticas disponíveis para abordar essa questão, com o início da geometria diferencial e da análise funcional moderna, evolução essa que pode ser ilustrada mencionando nomes tais como Élie Cartan e Hassler Whitney (que inventaram, respectivamente, os conceitos de formas diferenciais e de variedades diferenciáveis) ou John von Neumann (que no seu livro estabeleceu os fundamentos matemáticos da mecânica quântica). Lançando mão desta terminologia moderna, podemos resumir a definição do espaço de fase de um sistema físico na mecânica clássica e na mecânica quântica: ambos partem de um espaço de configuração que é uma variedade $Q$ descrevendo o conjunto de todas as posições possíveis das partículas que compõem o sistema, sendo que o espaço de fase na mecânica clássica é o fibrado cotangente $T^{*} Q$ de $Q$, enquanto que o espaço de fase na mecânica quântica é o espaço projetivo associado ao espaço de Hilbert $L^{2}(Q)$ das funções quadraticamente integráveis sobre $Q$.

No entanto, houve um pequeno problema com esta regra geral, a saber, não foi possível incorporar nela o fenômeno do spin das partículas que compõem todo o nosso mundo: pró- 
tons, elétrons e nêutrons. Na mecânica quântica, a modificação necessária para acomodar este fenômeno é simples: para partículas de spin $s(s=0,1 / 2,1, \ldots)$, usa-se $L^{2}\left(Q, \mathbb{C}^{2 s+1}\right)$ ao invés de $L^{2}(Q)$. Mas na mecânica clássica, as tentativas de acomodar o mesmo fenômeno pela mera substituição do espaço de configuração original por algum outro fracassaram, levando alguns autores de livros de Física à afirmação de que o spin seria um efeito puramente quântico. Mas nos anos 60 do século 20, Souriau mostrou que esta afirmação estava errada e que o que precisava ser feito era substituir não $Q$ e $\operatorname{sim} T^{*} Q$ : o problema foi resolvido usando $T^{*} Q \times S^{2}$, onde $S^{2}$ é a "esfera de Souriau" (cujo raio depende do spin $s$ ), ao invés de $T^{*} Q$. Com isso, Souriau alterou o paradigma da mecânica clássica, no sentido de que o espaço de fase de um sistema na mecânica clássica passa a ser uma variedade simplética, que pode ser um fibrado cotangente, ou não.

Em teoria de campos, a história é um pouco diferente e até hoje não há uma solução definitiva, pois aqui o fato de estarmos lidando com sistemas com um número infinito de graus de liberdade introduz uma complicação adicional e, pelo menos no âmbito quântico, não superada até hoje. E mesmo no âmbito clássico, trata-se de um problema difícil, pois aqui o espaço de fase é uma "variedade" de dimensão infinita: a variante mais elegante é o espaço de fase covariante, que é o espaço de todas as soluções das equações de movimento e que, mediante escolha (não covariante) de uma hipersuperfície de Cauchy no espaço-tempo, pode ser identificado com o espaço de todos os possíveis dados iniciais para o problema de Cauchy. Porém, em teoria de campos existe, pelo menos no caso relativístico, um princípio físico alheio à mecânica que serve como remédio para reduzir todas as construções necessárias em dimensão infinita, que genericamente são intratáveis, a construções em dimensão finita; é o princípio de localidade no espaço-tempo. Por exemplo, a dinâmica de sistemas com um número infinito de graus de liberdade é regida por equações de movimento que, genericamente, são equações funcionais, enquanto que em teoria de campos, são equações diferenciais (parciais) de uma determinada ordem. Além disso, quase todas as equações de movimento da teoria de campos que aparecem em modelos importantes são equações de (no máximo) segunda ordem e são equações de Euler-Lagrange associadas a um princípio variacional, admitindo tanto uma formulação lagrangiana como uma formulação hamiltoniana. E é um princípio variacional de primeira ordem, no sentido de que a Lagrangiana ou Hamiltoniana subjacente (em cada ponto do espaço-tempo) depende apenas dos valores, neste ponto, dos campos e das suas primeiras derivadas (no caso lagrangiano) ou dos campos e dos multimomentos conjugados às suas primeiras derivadas (no caso hamiltoniano). Ademais, tais sistemas lagrangianos/hamiltonianos de primeira ordem podem ser tratados exclusivamente no âmbito de variedades e fibrados de dimensão finita, eliminando grande parte das dificuldades que surgem na geometria diferencial em dimensão infinita. A versão hamiltoniana deste tratamento é conhecida como o formalismo multissimplético, cujas origens remontam a trabalhos de Caratheodory e Weyl nos anos 30 do século 20, mas cuja verdadeira natureza matemática foi desvendada apenas a partir dos anos 90 do mesmo século.

Posto isso, podemos nos perguntar se e como os métodos do formalismo multisimplético podem ser estendidos para se tornarem aplicáveis a sistemas lagrangianos/ hamiltonianos de ordem superior $(>1)$. O motivo mais importante para estudar essa questão, além do desejo da generalização por si só, reside na observação de que existe pelo menos uma teoria de importância fundamental e inegável para a Física que foge do 
padrão da primeira ordem: é a relatividade geral, cuja Lagrangiana é a curvatura escalar, a qual depende da métrica e suas derivadas parciais até segunda ordem. Porém, deve-se ressaltar que existe uma alternativa. Mesmo a relatividade geral pode ser enquadrada no formalismo de primeira ordem se lançarmos mão do formalismo de Palatini, no qual os campos fundamentais são a métrica e a conexão, de modo que a lagrangiana se torna uma lagrangiana de primeira ordem e a relação de Levi-Civita entre métrica e conexão é reestabelecida como parte das equações de movimento, na forma de um vínculo. Além disso, é sabido que tanto o formalismo de Poincaré-Cartan ([10]) quanto o formalismo de Hamilton-Cartan ([11], [12]) contemplam apenas jatos de ordem $2 r$ - 1, i.e, não contemplam fibrados de jatos de segunda ordem. Ainda assim, o assunto desse trabalho continua sendo relevante por investigar se as definições modernas de estruturas multissimpléticas e polissimpléticas estão em sintonia com esses formalismos.

O presente trabalho se divide da seguinte forma. No Capítulo 1, introduzimos formalmente fibrados gerais e a construção dos correspondentes fibrados de jatos de primeira e segunda ordem, juntamente com a dos fibrados duais, chamados de fibrados de cojatos. No Capítulo 2, definimos estruturas simpléticas sobre variedades e suas generalizações, as estruturas multissimpléticas e polissimpléticas, seguindo a construção em [8]. No Capítulo 3, combinamos os conceitos introduzidos nos capítulos anteriores, mostrando como as estruturas apresentadas no Capítulo 2 aparecem em fibrados de cojatos como os considerados no Capítulo 1. Aqui, consideramos não apenas o caso padrão de (co)jatos de primeira ordem, mas também o de (co)jatos de segunda ordem, que não parece ter sido tratado anteriormente na literatura. Dessa forma, procuramos evidenciar os desafios, intrínsecos tanto à mecânica clássica quanto à teoria de campos, de se definir uma estrutura multissimplética no dual de um fibrado de jatos holônomos de segunda ordem. 



\section{Capítulo 1}

\section{Fibrados de Jatos e Cojatos}

Antes de iniciarmos, é conveniente que façamos um apanhado de definições que utilizaremos ao longo do trabalho. A seguir, definiremos espaço afim, conexões e fibrado afim antes de iniciarmos o assunto de jatos propriamente dito.

\subsection{Preliminares Algébricos}

Sejam $K$ um corpo e $V$ um espaço vetorial sobre $K$. Um espaço afim modelado por $V$ é um conjunto $A$ junto com um mapa

$$
\begin{aligned}
+: A \times V & \rightarrow A \\
(a, v) & \mapsto a+v,
\end{aligned}
$$

chamado soma, que satisfaça:

- Se 0 é o elemento neutro aditivo de $V, a+0=a$ para todo $a \in A$;

- $a+(u+v)=(a+u)+v$ para todo $a \in A$ e todo $u, v \in V$

- Dados $a, b \in A$, existe um único $v \in V$ tal que $a+v=b$.

Tal mapa + configura uma ação transitiva simples de $V$ sobre $A$, na qual $V$ é considerado como grupo aditivo, sendo que "simples" é sinônimo de "sem ponto fixo". Fixando um vetor $v \in V$, o mapa $A \rightarrow A, a \mapsto a+v$, é chamado a translação por $v$. Note que uma vez que a ação do grupo aditivo é transitiva simples, existe um mapa bem definido

$$
\begin{aligned}
-: A \times A & \rightarrow V \\
(b, a) & \mapsto b-a
\end{aligned}
$$

chamado diferença, o que nos motiva chamar $V$ o espaço vetorial de diferenças de $A$ ([1], [2], [5]).

Em muitos casos, constrói-se espaços afins como subespaços de espaços vetoriais. Por exemplo, dados dois espaços vetoriais $W$ e $X$ e um mapa linear fixo $T: W \rightarrow X$, podemos 
definir $V=\operatorname{ker} T$ e, escolhendo qualquer ponto $x$ fixo em $X, A=\{w \in W: T w=x\}$. Um exemplo específico desta construção segue.

Considere uma sequência exata de espaços vetoriais de dimensão finita:

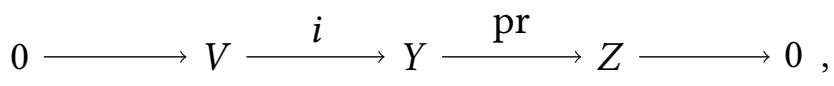

i.e, $i$ é uma injeção, pr é uma sobrejeção e ker pr $=\operatorname{im} i$. Tome $W=L(Z, Y), X=L(Z, Z)$ e defina o mapa linear $T: W \rightarrow X$ por $u \mapsto$ pr॰ $u$ para cada $u \in L(Z, Y)$. Então ker $T=L(Z, V)$. Além disso, se $x \in L(Z, Z)$ é um elemento fixo, $\{\sigma \in L(Z, Y): \operatorname{pr} \circ \sigma=x\}$ é um espaço afim modelado por $L(Z, V)$. Em particular, quando $x=\mathrm{id}_{Z}$, chamamos os elementos do espaço afim $\Sigma=\left\{\sigma \in L(Z, Y): \operatorname{pr} \circ \sigma=\mathrm{id}_{Z}\right\}$ de seções de pr.

Fixando uma seção $\sigma$, note que $\sigma \circ p r$ é uma projeção, ou seja, $(\sigma \circ p r)^{2}=\sigma \circ p$. Portanto $Y$ se decompõe na soma direta do seu núcleo com a sua imagem. Dizemos que o mapa $\sigma$, também chamado de levantamento horizontal, cinde o espaço $Y$ na soma direta $Y=\operatorname{ker} \operatorname{pr} \oplus \operatorname{im} \sigma$. Chamamos $V=\operatorname{ker} \operatorname{pr}$ de espaço de vetores verticais de $Y$ com projeção vertical $\operatorname{id}_{Y}-\sigma \circ$ pr e $H=\operatorname{im} \sigma$ de espaço de vetores horizontais de $Y$ com projeção $\sigma \circ p r$. Esse cenário configura a versão algébrica de conexão.

\subsection{Fibrados}

Dadas duas variedades $M$ e $Q$, o exemplo mais elementar de fibrado é o produto cartesiano $M \times Q$, no qual existe uma assimetria intrínseca entre os dois fatores expressa pela projeção $\mathrm{pr}_{1}: M \times Q \rightarrow M$, que na linguagem da Física corresponde à distinção entre variáveis dependentes e independentes. Uma situação mais geral e muito importante em aplicações ocorre quando o produto cartesiano é substituido por uma variedade $E$ que seja pelo menos "localmente" difeomorfa a $M \times Q$. Para formalizar esta ideia, define-se um fibrado como uma tripla $(E, \pi, M)$, na qual $M$ e $E$ são variedades chamadas respectivamente de base e espaço total e $\pi: E \rightarrow M$ é uma submersão sobrejetora chamada de projeção, satisfazendo a condição de trivialidade local: existem uma variedade $Q$, chamada de fíbra típica, um recobrimento aberto $\left(U_{\alpha}\right)_{\alpha \in A}$ de $M$ e uma família $\left(\Phi_{\alpha}\right)_{\alpha \in A}$ de difeomorfismos

$$
\Phi_{\alpha}: \pi^{-1}\left(U_{\alpha}\right) \rightarrow U_{\alpha} \times Q
$$

chamados de trivializações locais, tais que para quaisquer $\alpha, \beta \in A$ com $U_{\alpha} \cap U_{\beta} \neq \varnothing, \Phi_{\alpha} \circ \Phi_{\beta}^{-1}$ é um difeomorfismo e $\operatorname{pr}_{1} \circ \Phi_{\alpha}=\pi$ para cada $\alpha \in A$.

Essa estrutura de produto local nos permite introduzir sistemas de coordenadas sobre o espaço total chamadas de coordenadas adaptadas. Tais coordenadas $\left(x^{\mu}, q^{i}\right)$, nas quais a projeção do fibrado se dá simplesmente pela omissão das coordenadas $q^{i}$, são construídas a partir de um sistema de coordenadas locais $x^{\mu}$ da variedade base $M$ sobre um aberto $U$, um sistema de coordenadas locais $q^{i}$ da fibra típica $Q$ e uma trivialização sobre $U$. Se passarmos a um novo sistema de coordenadas adaptadas $\left(x^{\prime v}, q^{\prime j}\right)$, a lei de transformação é restrita pela condição de que as $x^{\prime \nu}$ são funções apenas das $x^{\mu}$ enquanto que as $q^{j}$ são funções tanto das $x^{\mu}$ quanto das $q^{i}$ ([3], [9]). 


\subsection{Jatos e Cojatos de Primeira Ordem}

Tome um fibrado $(E, \pi, M)$ e seja $e \in E$ tal que $\pi(e)=x$ em $M$. Suponha que $v$ pertença a $T_{e} E$, o espaço tangente a $E$ no ponto $e$. Dizemos que $v$ é vertical se $T_{e} \pi(v)=0$. Denotamos o espaço tangente de vetores verticais sobre $e$ por $V_{e} E$, que é o espaço tangente à fibra $\pi^{-1}(x)$ no ponto $e$. Isso nos revela a seguinte sequência exata de espaços vetoriais de dimensões finitas:

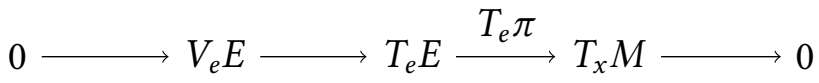

O conjunto $\Sigma_{e}$ de seções de $T_{e} \pi$ na dada sequência é um espaço afim, como já vimos, e é dita espaço afim de jatos em $e$. Denotaremos este espaço por $J_{e} E$. O espaço de diferenças de $J_{e} E$ é o espaço vetorial $V_{e} E \otimes T_{x}^{*} M$, que é dito espaço de jatos lineares e denotado por $\vec{J}_{e} E$. A união desses espaços $\bigcup_{e \in E} J_{e} E=J E$ e $\bigcup_{e \in E} \vec{J}_{e} E=\vec{J} E$ dão origem a dois fibrados sobre $E$, um afim e outro vetorial, com projeções-alvo (do inglês, target)

$$
\pi_{1,0}: J E \rightarrow E \quad \vec{\pi}_{1,0}: \vec{J} E \rightarrow E,
$$

e também a dois fibrados sobre $M$ com projeções-fonte (do inglês, source)

$$
\pi_{1}=\pi \circ \pi_{1,0} \quad \vec{\pi}_{1}=\pi \circ \vec{\pi}_{1,0} .
$$

Dados dois fibrados $\left(E, \pi_{E}, M\right)$ e $\left(F, \pi_{F}, M\right)$ e um homomorfismo estrito $f: E \rightarrow F$, podemos associar a eles um homomorfismo de fibrados afins $J f: J E \rightarrow J F$ e um homomorfismo de fibrados vetoriais $\vec{J} f: \vec{J} E \rightarrow \vec{J} F$ que recobrem $f$. Do ponto de vista de fibrados sobre $M$, podemos dizer que de fato $J f$ e $\vec{J} f$ são homomorfismos estritos de fibrados (já que recobrem a identidade de $M$ ), o que faz de $J$ e $\vec{J}$ funtores na categoria de fibrados sobre uma variedade base fixa ([7]). Para mostrar como esses funtores agem, tome $e \in E$ com projeção $\pi(e)=x$ e $u \in J_{e} E$ com projeção $\pi_{E}(u)=e$. O homomorfismo estrito $f$ implica que $\pi_{E}(e)=\left(\pi_{F} \circ f\right)(e)$. Logo, $T_{e} \pi_{E} \circ u=T_{f(e)} \pi_{F} \circ T_{e} f \circ u$, no qual denotamos

$$
T_{e} f \circ u=J_{e} f(u) .
$$

Analogamente,

$$
T_{e} f \circ \vec{u}=\vec{J}_{e} f(\vec{u})
$$

em que $\vec{u} \in \vec{J}_{e} E$.

Agora, dada uma seção $\varphi$ de $\pi_{E}$, definimos uma seção $\psi$ de $\pi_{F}$ como $f \circ \varphi=\psi$. Logo, $T f \circ T \varphi=T \psi$. Note que $T_{x} \psi \in L\left(T_{x} M, T_{\varphi(x)} E\right)$ e $T_{x}\left(\pi_{F} \circ \psi\right)=\operatorname{id}_{T_{x} M}$ para todo $x \in M$ no domínio de $\psi$. Portanto, $T \psi$ é uma seção de $T \pi_{F}$. Chamaremos esta seção de extensão por jato de $\psi$, denotando-a por $j \psi$. Assim, temos que

$$
j(f \circ \varphi)=J f \circ j \varphi .
$$

As equações (1.1), (1.2) e (1.3) evidenciam que o comportamento de $J f$ e $\vec{J} f$ se dá através da tangente de $f$ e que a extensão $j \varphi$ de $\varphi$ é uma reinterpretação do mapa tangente de $\varphi$, ou seja, a extensão (de primeira ordem) por jato de uma seção carrega consigo informações 
da seção original e da primeira derivada desta. Apesar disto, é importante observar que, dada uma seção $\tilde{\varphi}$ de $\pi_{1}$, não é automática a existência de uma seção $\varphi$ de $\pi$ tal que $j \varphi=\tilde{\varphi}$. As seções de $\pi_{1}$ que satisfazem essa condição são chamadas de holônomas. Esta definição se estenderá naturalmente a jatos de ordem mais alta. Entretanto, note que, para qualquer $u \in J_{e} E$, existe uma seção $\varphi$ de $\pi$ tal que $j_{x} \varphi=u$. Isto segue diretamente da definição de vetor tangente a uma dada variedade.

Tome $\left(x^{\mu}, q^{i}\right)$ um sistema de coordenadas adaptadas em $E$. Como vimos, se $\pi(e)=$ $x, u \in L\left(T_{x} M, T_{e} E\right)$ é um elemento do espaço afim de jatos $J_{e} E$ se, e somente se, $T_{e} \pi \circ u=\mathrm{id}_{T_{x} M}$, e, de modo semelhante, $\vec{u} \in L\left(T_{x} M, T_{e} E\right)$ é um elemento do espaço vetorial de jatos linearizados $\vec{J}_{e} E$ se, e somente se, vale $T_{e} \pi \circ \vec{u}=0$. Em particular, a imagem de $u$ é um subespaço vetorial $H_{e} E \subset T_{e} E$ complementar ao subespaço vertical $V_{e} E$, que podemos chamar de espaço horizonal: $H_{e} E \oplus V_{e} E=T_{e} E$. Assim, podemos escrever

$$
u=\left(\frac{\partial}{\partial x^{\mu}}+q_{\mu}^{i}(u) \frac{\partial}{\partial q^{i}}\right) \otimes d x^{\mu} \quad \vec{u}=\vec{q}_{\mu}^{i}(\vec{u}) \frac{\partial}{\partial q^{i}} \otimes d x^{\mu},
$$

de modo que as $q_{\mu}^{i}$ e $\vec{q}_{\mu}^{i}$ fazem parte de um sistema de coordenadas induzidas $\left(x^{\mu}, q^{i}, q_{\mu}^{i}\right)$ no espaço total $J E$ e $\left(x^{\mu}, q^{i}, \vec{q}_{\mu}^{i}\right)$ no espaço total $\vec{J} E$. Se passarmos para um novo sistema de coordenadas adaptadas $\left(x^{\prime v}, q^{\prime j}\right)$, teremos

$$
\frac{\partial}{\partial x^{\mu}}=\frac{\partial x^{\prime v}}{\partial x^{\mu}} \frac{\partial}{\partial x^{\prime v}}+\frac{\partial q^{j}}{\partial x^{\mu}} \frac{\partial}{\partial q^{\prime j}} \quad d x^{\mu}=\frac{\partial x^{\mu}}{\partial x^{\prime v}} d x^{\prime v} \quad \frac{\partial}{\partial q^{i}}=\frac{\partial q^{j}}{\partial q^{i}} \frac{\partial}{\partial q^{\prime j}}
$$

e, portanto, a lei de transformação para as coordenadas induzidas será a seguinte:

$$
q_{v}^{\prime j}=\frac{\partial q^{\prime j}}{\partial q^{i}} \frac{\partial x^{\mu}}{\partial x^{\prime \nu}} q_{\mu}^{i}+\frac{\partial q^{\prime j}}{\partial x^{\mu}} \frac{\partial x^{\mu}}{\partial x^{\prime v}} \quad \vec{q}_{v}^{\prime j}=\frac{\partial q^{j}}{\partial q^{i}} \frac{\partial x^{\mu}}{\partial x^{\prime \nu}} \vec{q}_{\mu}^{i}
$$

Passando a duais, considere o espaço dual ao espaço afim $J_{e} E$, definido por

$$
J_{e}^{\star} E=\left\{z: J_{e} E \rightarrow \mathbb{R} \mid z \text { é afim }\right\},
$$

cujo espaço vetorial associado é o espaço dual ao espaço vetorial $\vec{J}_{e} E$, i.e,

$$
\vec{J}_{e}^{\star} E=\left\{\vec{z}: \vec{J}_{e} E \rightarrow \mathbb{R} \mid \vec{z} \text { é linear }\right\} .
$$

Entretanto, os espaços de fase na teoria de campos são construídos a partir da substituição da reta real pelo espaço unidimensional de formas de volume sobre a variedade base $M$ ponto a ponto. Em outras palavras, dado um ponto $e$ de $E$ de projeção $\pi(e)=x \operatorname{em} M$, definimos o dual afim torcido $J_{e}^{\circledast} E$ de $J_{e} E$ e o dual linear torcido $\vec{J}_{e}^{\oplus} E$ de $\vec{J}_{e} E$ como

$$
J_{e}^{\circledast} E=\left\{z: J_{e} E \rightarrow \bigwedge^{n} T_{x}^{*} M \mid z \text { é afim }\right\},
$$

$\mathrm{e}$

$$
\vec{J}_{e}^{\circledast} E=\left\{\vec{z}: \vec{J}_{e} E \rightarrow \bigwedge^{n} T_{x}^{*} M \mid \vec{z} \text { é linear }\right\} .
$$

Tomando as uniões disjuntas enquanto $e$ percorre $E$, definimos os fibrados $J^{\star} E, \vec{J}^{\star} E, J^{\circledast} E$ e $\vec{J}^{\oplus} E$ de duas formas possíveis: cada um deles é um fibrado sobre $M$ com respeito à 
projeção-fonte e um fibrado vetorial sobre $E$ com respeito à projeção-alvo. Nos referimos a todos esses fibrados como fibrados de cojatos. Enquanto fibrados vetoriais sobre $E$, temos que

$$
J^{\circledast} E=J^{\star} E \otimes \pi^{*}\left(\bigwedge^{n} T^{*} M\right),
$$

$\mathrm{e}$

$$
\vec{J}^{\oplus} E=\vec{J}^{*} E \otimes \pi^{*}\left(\bigwedge^{n} T^{*} M\right) .
$$

Além disso, $J^{\star} E$ é um fibrado afim sobre $\overrightarrow{J^{\star}} E$ e, analogamente, $J^{\circledast} E$ é um fibrado afim sobre $\vec{J}^{\oplus} E$. Os elementos desses espaços são definidos através do pareamento com os elementos de $J E$ de forma que

$$
\langle z, u\rangle=p_{i}^{\mu} q_{\mu}^{i}+p \quad\langle\vec{z}, \vec{u}\rangle=p_{i}^{\mu} \vec{q}_{\mu}^{i}
$$

e, no caso torcido,

$$
\langle z, u\rangle=\left(p_{i}^{\mu} q_{\mu}^{i}+p\right) d^{n} x \quad\langle\vec{z}, \vec{u}\rangle=\left(p_{i}^{\mu} \vec{q}_{\mu}^{i}\right) d^{n} x .
$$

Se passarmos para um novo sistema de coordenadas adaptadas $\left(x^{\prime v}, q^{\prime j}\right)$, teremos

$$
p_{i}^{\mu} q_{\mu}^{i}+p=p_{j}^{\prime v} q_{v}^{\prime j}+p^{\prime} \quad p_{i}^{\mu} \vec{q}_{\mu}^{i}=p_{j}^{\prime v} \vec{q}_{v}^{\prime j}
$$

e, portanto, a lei de transformação para as coordenadas induzidas de $J^{\star} E$ será a seguinte:

$$
p_{j}^{\prime \nu}=\frac{\partial q^{i}}{\partial q^{\prime j}} \frac{\partial x^{\prime \nu}}{\partial x^{\mu}} p_{i}^{\mu} \quad p^{\prime}=p-\frac{\partial q^{i}}{\partial q^{\prime j}} \frac{\partial q^{\prime j}}{\partial x^{\mu}} p_{i}^{\mu}
$$

enquanto que a lei de transformação para as coordenadas induzidas de $J^{\circledast} E$ será a seguinte:

$$
p_{j}^{\prime v}=\operatorname{det}\left(\frac{\partial x}{\partial x^{\prime}}\right) \frac{\partial q^{i}}{\partial q^{\prime j}} \frac{\partial x^{\prime \nu}}{\partial x^{\mu}} p_{i}^{\mu} \quad p^{\prime}=\operatorname{det}\left(\frac{\partial x}{\partial x^{\prime}}\right)\left(p-\frac{\partial q^{i}}{\partial q^{\prime j}} \frac{\partial q^{j}}{\partial x^{\mu}} p_{i}^{\mu}\right)
$$

\subsection{Jatos e Cojatos de Segunda Ordem}

Tome a seguinte sequência exata sobre $\left(J E, \pi_{1}, M\right)$ :

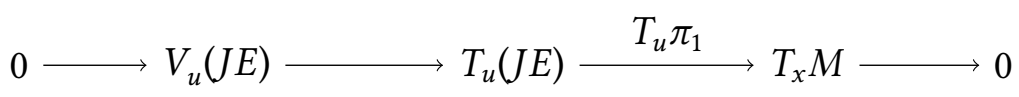

no qual $u \in J E$ e $\pi_{1}(u)=x$.

Analogamente ao espaço de jatos de primeira ordem, o conjunto de seções de $T_{u} \pi_{1}$ na dada sequência é um espaço afim e é dito espaço afim de jatos iterados em $u$. Denotaremos este espaço por $J_{u}(J E)$. O espaço de diferenças de $J_{u}(J E)$ é o espaço vetorial $V_{u}(J E) \otimes T_{x}^{*} M$, que é dito espaço de jatos lineares iterados em $u$ e denotado por $\vec{J}_{u}(J E)$. Assim como na primeira ordem, as uniões $\bigcup_{u \in J E} J_{u}(J E)=J(J E)$ e $\bigcup_{u \in J E} \vec{J}_{u}(J E)=\vec{J}(J E)$ dão origem a dois 
fibrados sobre $J E$, um afim e outro vetorial, com projeções-alvo

$$
\pi_{2,1}: J(J E) \rightarrow J E \quad \vec{\pi}_{2,1}: \vec{J}(J E) \rightarrow J E,
$$

e também a dois fibrados sobre $M$ com projeções-fonte

$$
\pi_{2}=\pi_{1} \circ \pi_{2,1} \quad \vec{\pi}_{2}=\pi_{1} \circ \vec{\pi}_{2,1} .
$$

Assuma as coordenadas adaptadas $\left(x^{\mu}, q^{i}\right)$ em $E$. Como foi visto, se $\pi_{1}(u)=x$, $w \in L\left(T_{x} M, T_{u}(J E)\right)$ é um elemento do espaço afim de jatos $J_{u}(J E)$ se, e somente se, $T_{u} \pi_{1} \circ w=\operatorname{id}_{T_{x} M}$, e de modo semelhante, $\vec{w} \in L\left(T_{x} M, T_{u}(J E)\right)$ é um elemento do espaço vetorial de jatos linearizados $\vec{J}_{u}(J E)$ se, e somente se, vale $T_{u} \pi_{1} \circ \vec{w}=0$. Em particular, a imagem de $w$ é um subespaço vetorial $H_{u}(J E) \subset T_{u}(J E)$ complementar ao subespaço vertical $V_{u}(J E)$, que podemos chamar de espaço horizontal: $H_{u}(J E) \oplus V_{u}(J E)=T_{u}(J E)$. Assim, podemos escrever,

$$
w=\left(\frac{\partial}{\partial x^{\mu}}+q_{; \mu}^{i}(w) \frac{\partial}{\partial q^{i}}+q_{\mu ; v}^{i}(w) \frac{\partial}{\partial q_{v}^{i}}\right) \otimes d x^{\mu} \quad \vec{w}=\vec{q}_{\mu ; \nu}^{i}(\vec{w}) \frac{\partial}{\partial q_{v}^{i}} \otimes d x^{\mu},
$$

de modo a induzir o sistema de coordenadas $\left(x^{\mu}, q^{i}, q_{\mu}^{i}, q_{; \mu}^{i}, q_{\mu ; \nu}^{i}\right)$ no espaço total $J(J E)$ e $\left(x^{\mu}, q^{i}, q_{\mu}^{i}, q_{; \mu}^{i}, \vec{q}_{\mu ; \nu}^{i}\right)$ no espaço total $\vec{J}(J E)$. Se passarmos para um novo sistema de coordenadas adaptadas $\left(x^{\prime v}, q^{\prime j}\right)$, teremos

$$
\begin{aligned}
\frac{\partial}{\partial x^{\mu}} & =\frac{\partial x^{\prime \alpha}}{\partial x^{\mu}} \frac{\partial}{\partial x^{\prime \alpha}}+\frac{\partial q^{\prime j}}{\partial x^{\mu}} \frac{\partial}{\partial q^{\prime j}}+\frac{\partial q_{\alpha}^{\prime j}}{\partial x^{\mu}} \frac{\partial}{\partial q_{\alpha}^{\prime j}} & d x^{\mu} & =\frac{\partial x^{\mu}}{\partial x^{\prime \alpha}} d x^{\prime \alpha} \\
\frac{\partial}{\partial q^{i}} & =\frac{\partial q^{\prime j}}{\partial q^{i}} \frac{\partial}{\partial q^{\prime j}}+\frac{\partial q_{\alpha}^{\prime j}}{\partial q^{i}} \frac{\partial}{\partial q_{\alpha}^{\prime j}} & \frac{\partial}{\partial q_{\mu}^{i}} & =\frac{\partial q_{\alpha}^{j}}{\partial q_{\mu}^{i}} \frac{\partial}{\partial q_{\alpha}^{\prime j}}
\end{aligned}
$$

e, considerando a equação (1.6) e a identidade

$$
\frac{\partial^{2} x^{\nu}}{\partial x^{\prime \rho} \partial x^{\prime \beta}}=-\frac{\partial x^{\sigma}}{\partial x^{\prime \rho}} \frac{\partial x^{\mu}}{\partial x^{\prime \beta}} \frac{\partial x^{\nu}}{\partial x^{\prime \alpha}} \frac{\partial^{2} x^{\prime \alpha}}{\partial x^{\sigma} \partial x^{\mu}},
$$

a lei de transformação para as coordenadas induzidas [2] será a seguinte:

$$
\begin{aligned}
& q_{; v}^{\prime j}=\frac{\partial q^{j j}}{\partial q^{i}} \frac{\partial x^{\mu}}{\partial x^{\prime \nu}} q_{; \mu}^{i}+\frac{\partial q^{j j}}{\partial x^{\mu}} \frac{\partial x^{\mu}}{\partial x^{\prime \nu}} \\
& \vec{q}_{\alpha ; \beta}^{\prime j}=\frac{\partial q^{j}}{\partial q^{i}} \frac{\partial x^{\mu}}{\partial x^{\prime \alpha}} \frac{\partial x^{v}}{\partial x^{\prime \beta}} \vec{q}_{\mu ; \nu}^{i} \\
& q_{\alpha ; \beta}^{j}=\frac{\partial q^{j}}{\partial q^{i}} \frac{\partial x^{\mu}}{\partial x^{\prime \alpha}} \frac{\partial x^{v}}{\partial x^{\prime \beta}} q_{\mu ; v}^{i}+\frac{\partial^{2} q^{j}}{\partial q^{i} \partial q^{k}} \frac{\partial x^{\mu}}{\partial x^{\prime \alpha}} \frac{\partial x^{v}}{\partial x^{\prime \beta}} q_{\mu}^{i} q_{; v}^{k} \\
& +\frac{\partial^{2} q^{j}}{\partial q^{k} \partial x^{\mu}} \frac{\partial x^{\mu}}{\partial x^{\prime \alpha}} \frac{\partial x^{v}}{\partial x^{\prime \beta}} q_{; \nu}^{k}+\left(\frac{\partial^{2} q^{j}}{\partial q^{i} \partial x^{v}} \frac{\partial x^{\mu}}{\partial x^{\prime \alpha}} \frac{\partial x^{v}}{\partial x^{\prime \beta}}-\frac{\partial^{2} x^{\prime \gamma}}{\partial x^{\kappa} \partial x^{\lambda}} \frac{\partial q^{j}}{\partial q^{i}} \frac{\partial x^{\mu}}{\partial x^{\prime \gamma}} \frac{\partial x^{\kappa}}{\partial x^{\prime \alpha}} \frac{\partial x^{\lambda}}{\partial x^{\prime \beta}}\right) q_{\mu}^{i} \\
& +\frac{\partial^{2} q^{\prime j}}{\partial x^{\mu} \partial x^{v}} \frac{\partial x^{\mu}}{\partial x^{\prime \alpha}} \frac{\partial x^{\nu}}{\partial x^{\prime \beta}}-\frac{\partial^{2} x^{\prime \gamma}}{\partial x^{\kappa} \partial x^{\lambda}} \frac{\partial q^{j}}{\partial x^{\mu}} \frac{\partial x^{\mu}}{\partial x^{\prime \gamma}} \frac{\partial x^{\kappa}}{\partial x^{\prime \alpha}} \frac{\partial x^{\lambda}}{\partial x^{\prime \beta}},
\end{aligned}
$$


Agora, note que $J(J E)$ (analogamente, $\vec{J}(J E)$ ) admite duas projeções sobre $J E$, a saber, $\pi_{2,1}$ e $J \pi_{1,0}$ (analogamente, $\vec{\pi}_{2,1}$ e $\left.\vec{J} \pi_{1,0}\right)$; em coordenadas, essas projeções levam $\left(x^{\mu}, q^{i}, q_{\mu}^{i}, q_{; \mu}^{i}, q_{\mu, v}^{i}\right)$ (analogamente, $\left.\left(x^{\mu}, q^{i}, q_{\mu}^{i}, q_{; \mu}^{i} \vec{q}_{\mu ; v}^{i}\right)\right)$ para $\left(x^{\mu}, q^{i}, q_{\mu}^{i}\right)$ e para $\left(x^{\mu}, q^{i}, q_{; \mu}^{i}\right)$, respectivamente. Define-se o fibrado $\bar{J}^{2} E$ de jatos semi-holônomos (analogamente, o fibrado $\overrightarrow{\bar{J}}^{2} E$ de jatos lineares semi-holônomos) de segunda ordem como o subfibrado de $J(J E)$ (analogamente, de $\vec{J}(J E)$ ) onde essas duas projeções coincidem, i.e,

$$
\bar{J}^{2} E=\left\{\bar{w} \in J(J E) \mid \pi_{2,1} \bar{w}=J \pi_{1,0} \bar{w}\right\} \quad \overrightarrow{\bar{J}}^{2} E=\left\{\overrightarrow{\underline{w}} \in \vec{J}(J E) \mid \vec{\pi}_{2,1} \overrightarrow{\bar{w}}=\vec{J} \pi_{1,0} \overrightarrow{\underline{w}}\right\} .
$$

O espaço total $\bar{J}^{2} E$ assume coordenadas $\left(x^{\mu}, q^{i}, q_{\mu}^{i}, q_{\mu, v}^{i}\right)$ e o espaço total $\overrightarrow{\vec{J}}^{2} E,\left(x^{\mu}, q^{i}, q_{\mu}^{i}, \vec{q}_{\mu, v}^{i}\right)$, sendo o primeiro um fibrado afim e o segundo seu fibrado vetorial de diferenças. Se passarmos novamente para um novo sistema de coordenadas adaptadas $\left(x^{\prime v}, q^{\prime j}\right)$, teremos as leis de transformação:

$$
\begin{aligned}
q_{v}^{\prime j}= & \frac{\partial q^{\prime j}}{\partial q^{i}} \frac{\partial x^{\mu}}{\partial x^{\prime \nu}} q_{\mu}^{i}+\frac{\partial q^{\prime j}}{\partial x^{\mu}} \frac{\partial x^{\mu}}{\partial x^{\prime v}} \\
\vec{q}_{\alpha ; \beta}^{j}= & \frac{\partial q^{\prime j}}{\partial q^{i}} \frac{\partial x^{\mu}}{\partial x^{\prime \alpha}} \frac{\partial x^{v}}{\partial x^{\prime \beta}} \vec{q}_{\mu ; v}^{i} \\
q_{\alpha ; \beta}^{\prime j}= & \frac{\partial q^{\prime j}}{\partial q^{i}} \frac{\partial x^{\mu}}{\partial x^{\prime \alpha}} \frac{\partial x^{v}}{\partial x^{\prime \beta}} q_{\mu ; v}^{i}+\frac{\partial^{2} q^{\prime j}}{\partial q^{i} \partial q^{k}} \frac{\partial x^{\mu}}{\partial x^{\prime \alpha}} \frac{\partial x^{v}}{\partial x^{\prime \beta}} q_{\mu}^{i} q_{v}^{k} \\
& +\left(\frac{\partial^{2} q^{\prime j}}{\partial q^{i} \partial x^{v}} \frac{\partial x^{v}}{\partial x^{\prime \alpha}} \frac{\partial x^{\mu}}{\partial x^{\prime \beta}}+\frac{\partial^{2} q^{\prime j}}{\partial q^{i} \partial x^{v}} \frac{\partial x^{\mu}}{\partial x^{\prime \alpha}} \frac{\partial x^{v}}{\partial x^{\prime \beta}}-\frac{\partial^{2} x^{\prime \gamma}}{\partial x^{k} \partial x^{\lambda}} \frac{\partial q^{\prime j}}{\partial q^{i}} \frac{\partial x^{\mu}}{\partial x^{\prime \gamma}} \frac{\partial x^{k}}{\partial x^{\prime \alpha}} \frac{\partial x^{\lambda}}{\partial x^{\prime \beta}}\right) q_{\mu}^{i} \\
& +\frac{\partial^{2} q^{\prime j}}{\partial x^{\mu} \partial x^{v}} \frac{\partial x^{\mu}}{\partial x^{\prime \alpha}} \frac{\partial x^{v}}{\partial x^{\prime \beta}}-\frac{\partial^{2} x^{\prime \gamma}}{\partial x^{\kappa} \partial x^{\lambda}} \frac{\partial q^{\prime j}}{\partial x^{\mu}} \frac{\partial x^{\mu}}{\partial x^{\prime \gamma}} \frac{\partial x^{k}}{\partial x^{\prime \alpha}} \frac{\partial x^{\lambda}}{\partial x^{\prime \beta}}
\end{aligned}
$$

Em um segundo passo, impõe-se a condição de simetria sobre as segundas derivadas parciais, que em coordenadas tomam as formas

$$
q_{\mu ; \nu}^{i}=q_{v ; \mu}^{i} \doteq q_{\mu v}^{i} \quad \vec{q}_{\mu ; \nu}^{i}=\vec{q}_{v ; \mu}^{i} \doteq \vec{q}_{\mu v}^{i}
$$

para chegar ao fibrado $J^{2} E$ de jatos holônomos (analogamente, ao fibrado $\vec{J}^{2} E$ de jatos lineares holônomos) de segunda ordem, sendo que, em relação à mesma projeção-alvo sobre $J E$, o primeiro é um fibrado afim e o segundo seu fibrado vetorial de diferenças.

Tomando as mesmas coordenadas adaptadas $\left(x^{\mu}, q^{i}\right)$ em $E$ como antes, $\pi_{1}(u)=x \mathrm{e}$ $w \in J^{2} E$, podemos escrever

$$
w=\left(\frac{\partial}{\partial x^{\mu}}+q_{\mu}^{i}(w) \frac{\partial}{\partial q^{i}}+q_{\mu \nu}^{i}(w) \frac{\partial}{\partial q_{v}^{i}}\right) \otimes d x^{\mu} \quad \vec{w}=\vec{q}_{\mu v}^{i}(\vec{w}) \frac{\partial}{\partial q_{v}^{i}} \otimes d x^{\mu} .
$$


Mais uma vez passando para um novo sistema de coordenadas adaptadas $\left(x^{\prime \alpha}, q^{\prime j}\right)$, temos a lei de transformação para as coordenadas induzidas:

$$
\begin{aligned}
q_{v}^{\prime j}= & \frac{\partial q^{\prime j}}{\partial q^{i}} \frac{\partial x^{\mu}}{\partial x^{\prime \nu}} q_{\mu}^{i}+\frac{\partial q^{\prime j}}{\partial x^{\mu}} \frac{\partial x^{\mu}}{\partial x^{\prime v}} \\
\vec{q}_{\alpha ; \beta}^{\prime j}= & \frac{\partial q^{\prime j}}{\partial q^{i}} \frac{\partial x^{\mu}}{\partial x^{\prime \alpha}} \frac{\partial x^{v}}{\partial x^{\prime \beta}} \vec{q}_{\mu ; \nu}^{i} \\
q_{\alpha \beta}^{\prime j}= & \frac{\partial q^{\prime j}}{\partial q^{i}} \frac{\partial x^{\mu}}{\partial x^{\prime \alpha}} \frac{\partial x^{v}}{\partial x^{\prime \beta}} q_{\mu v}^{i}+\frac{\partial^{2} q^{\prime j}}{\partial q^{i} \partial q^{k}} \frac{\partial x^{\mu}}{\partial x^{\prime \alpha}} \frac{\partial x^{v}}{\partial x^{\prime \beta}} q_{\mu}^{i} q_{v}^{k} \\
& +\left(\frac{\partial^{2} q^{\prime j}}{\partial q^{i} \partial x^{v}} \frac{\partial x^{v}}{\partial x^{\prime \alpha}} \frac{\partial x^{\mu}}{\partial x^{\prime \beta}}+\frac{\partial^{2} q^{\prime j}}{\partial q^{i} \partial x^{v}} \frac{\partial x^{\mu}}{\partial x^{\prime \alpha}} \frac{\partial x^{v}}{\partial x^{\prime \beta}}-\frac{\partial^{2} x^{\prime \gamma}}{\partial x^{\kappa} \partial x^{\lambda}} \frac{\partial q^{\prime j}}{\partial q^{i}} \frac{\partial x^{\mu}}{\partial x^{\prime \gamma}} \frac{\partial x^{\kappa}}{\partial x^{\prime \alpha}} \frac{\partial x^{\lambda}}{\partial x^{\prime \beta}}\right) q_{\mu}^{i} \\
& +\frac{\partial^{2} q^{\prime j}}{\partial x^{\mu} \partial x^{v}} \frac{\partial x^{\mu}}{\partial x^{\prime \alpha}} \frac{\partial x^{v}}{\partial x^{\prime \beta}}-\frac{\partial^{2} x^{\prime \gamma}}{\partial x^{\kappa} \partial x^{\lambda}} \frac{\partial q^{\prime j}}{\partial x^{\mu}} \frac{\partial x^{\mu}}{\partial x^{\prime \gamma}} \frac{\partial x^{\kappa}}{\partial x^{\prime \alpha}} \frac{\partial x^{\lambda}}{\partial x^{\prime \beta}},
\end{aligned}
$$

Passando a duais, tome $u \in J E$ e considere os espaços duais aos espaços afins $J_{u}^{2} E$ e $\bar{J}_{u}^{2} E$, definidos respectivamente por

$$
J_{u}^{2 \star} E=\left\{z: J_{u}^{2} E \rightarrow \mathbb{R} \mid z \text { é afim }\right\} \quad \bar{J}_{u}^{2 \star} E=\left\{\bar{z}: \bar{J}_{u}^{2} E \rightarrow \mathbb{R} \mid \bar{z} \text { é afim }\right\}
$$

cujos espaços vetoriais associados são os espaços duais aos respectivos espaços vetoriais $\vec{J}_{u}^{2} E$ e ${\overrightarrow{J_{u}}}_{u}^{2} E$, i.e,

$$
\vec{J}_{u}^{2 *} E=\left\{\vec{z}: \vec{J}_{u}^{2} E \rightarrow \mathbb{R} \mid \vec{z} \text { é linear }\right\} \quad{\overrightarrow{J_{u}}}_{u}^{2 \star} E=\left\{\overrightarrow{\vec{z}}:{\overrightarrow{J_{u}}}_{u}^{2} E \rightarrow \mathbb{R} \mid \overrightarrow{\bar{z}} \text { é linear }\right\} .
$$

Entretanto, os espaços de fase na teoria de campos são construídos a partir da substituição da reta real pelo espaço unidimensional de formas de volume sobre a variedade base $M$ ponto a ponto. Em outras palavras, dado um ponto $u$ de $J E$ de projeção $\pi_{1}(u)=x$ em $M$, definimos os duais afins torcidos $J_{u}^{2 \circledast} E$ de $J_{u}^{2} E$ e $\bar{J}_{u}^{2 \circledast} E$ de $\bar{J}_{u}^{2} E$, assim como os duais lineares torcidos $\vec{J}_{u}^{2 \circledast} E$ de $\vec{J}_{u}^{2} E$ e $\overrightarrow{\vec{J}}_{u}^{2 \circledast} E$ de $\overrightarrow{\vec{J}}_{u}^{2} E$, como

$$
J_{u}^{2 \circledast} E=\left\{z: J_{u}^{2} E \rightarrow \bigwedge^{n} T_{x}^{*} M \mid z \text { é afim }\right\} \quad \bar{J}_{u}^{2 \circledast} E=\left\{\bar{z}: \bar{J}_{u}^{2} E \rightarrow \bigwedge^{n} T_{x}^{*} M \mid \bar{z} \text { é afim }\right\}
$$

$\mathrm{e}$

$$
\vec{J}_{u}^{2 \oplus} E=\left\{\vec{z}: \vec{J}_{u}^{2} E \rightarrow \bigwedge^{n} T_{x}^{*} M \mid \vec{z} \text { é linear }\right\} \quad \overrightarrow{\bar{J}}_{u}^{2 \oplus} E=\left\{\overrightarrow{\vec{z}}: \overrightarrow{\bar{J}}_{u}^{2} E \rightarrow \bigwedge^{n} T_{x}^{*} M \mid \overrightarrow{\bar{z}} \text { é linear }\right\} .
$$

Tomando as uniões disjuntas enquanto $u$ percorre $J E$, definimos os fibrados $J^{2 \star} E, \vec{J}^{2 \star} E$, $J^{2 \circledast} E$ e $\vec{J}^{2 \circledast} E$ (analogamente, $\vec{J}^{2 \star} E, \vec{J}^{2 \star} E, \vec{J}^{2 \circledast} E$ e $\vec{J}^{2 \circledast} E$ ) de duas formas possíveis: cada um deles é um fibrado sobre $M$ com respeito à projeção-fonte e um fibrado vetorial sobre $J E$ (analogamente, sobre $\bar{J} E$ ) com respeito à projeção-alvo. Nos referimos a todos esses fibrados como fibrados de cojatos. Enquanto fibrados vetoriais sobre $J E$ e fibrados vetoriais sobre $\bar{J} E$, temos que

$$
J^{2 \circledast} E=J^{2 \star} E \otimes \pi_{1}^{*}\left(\bigwedge^{n} T^{\star} M\right) \quad \bar{J}^{2 \circledast} E=\bar{J}^{2 \star} E \otimes \pi_{1}^{*}\left(\bigwedge^{n} T^{\star} M\right)
$$


e

$$
\vec{J}^{2 \circledast} E=\vec{J}^{2 *} E \otimes \pi_{1}^{*}\left(\bigwedge^{n} T^{*} M\right) \quad \overrightarrow{\bar{J}}^{2 \circledast} E=\overrightarrow{\bar{J}}^{2 *} E \otimes \pi_{1}^{*}\left(\bigwedge^{n} T^{*} M\right) .
$$

Além disso, $J^{2 \star} E$ é um fibrado afim sobre $\vec{J}^{2 \star} E, \vec{J}^{2 \star} E$ é um fibrado afim sobre $\vec{J}^{2 \star} E$ e, analogamente, $J^{2 \circledast} E$ é um fibrado afim sobre $\vec{J}^{2 \circledast} E$ e $\vec{J}^{2 \circledast} E$ é um fibrado afim sobre $\vec{J}^{2 \circledast} E$. Assim, os elementos desses espaços são definidos através do pareamento com os respectivos elementos de $J^{2} E$ e de $\bar{J}^{2} E$ de forma que

$$
\begin{array}{ll}
\langle\bar{z}, \bar{w}\rangle=p_{i}^{\mu ; v} q_{\mu, v}^{i}+\bar{r} & \langle\overrightarrow{\bar{z}}, \overrightarrow{\bar{w}}\rangle=p_{i}^{\mu, v} \vec{q}_{\mu ; \nu}^{i} \\
\langle z, w\rangle=p_{i}^{\mu v} q_{\mu \nu}^{i}+r & \langle\vec{z}, \vec{w}\rangle=p_{i}^{\mu v} \vec{q}_{\mu \nu}^{i}
\end{array}
$$

e, no caso torcido,

$$
\begin{array}{ll}
\langle\bar{z}, \bar{w}\rangle=\left(p_{i}^{\mu, v} q_{\mu, v}^{i}+\bar{r}\right) d^{n} x & \langle\overrightarrow{\bar{z}}, \overrightarrow{\bar{w}}\rangle=\left(p_{i}^{\mu ; v} \vec{q}_{\mu, v}^{i}\right) d^{n} x \\
\langle z, w\rangle=\left(p_{i}^{\mu v} q_{\mu \nu}^{i v}+r\right) d^{n} x & \langle\vec{z}, \vec{w}\rangle=\left(p_{i}^{\mu v} \vec{q}_{\mu v}^{i}\right) d^{n} x .
\end{array}
$$

Se passarmos para um novo sistema de coordenadas adaptadas $\left(x^{\prime v}, q^{\prime j}\right)$, teremos respectivamente que

$$
\begin{aligned}
p_{i}^{\mu ; v} q_{\mu ; v}^{i}+\bar{r} & =p_{j}^{\prime \alpha ; \beta} q_{\alpha ; \beta}^{\prime j}+\bar{r}^{\prime} & p_{i}^{\mu ; v} \vec{q}_{\mu ; v}^{i} & =p_{j}^{\prime \alpha ; \beta} \vec{q}_{\alpha ; \beta}^{\prime j} \\
p_{i}^{\mu v} q_{\mu v}^{i}+r & =p_{j}^{\prime \alpha \beta} q_{\alpha \beta}^{\prime j}+r^{\prime} & p_{i}^{\mu v} \vec{q}_{\mu v}^{i} & =p_{j}^{\prime \alpha \beta} \vec{q}_{\alpha \beta}^{\prime j}
\end{aligned}
$$

e, portanto, a lei de transformação para as coordenadas induzidas de $\bar{J}^{2 \star} E$ será a seguinte:

$$
\begin{aligned}
p_{j}^{\prime \alpha ; \beta}= & \frac{\partial q^{i}}{\partial q^{\prime j}} \frac{\partial x^{\prime \alpha}}{\partial x^{\mu}} \frac{\partial x^{\prime \beta}}{\partial x^{v}} p_{i}^{\mu ; v} \\
\bar{r}^{\prime}= & \bar{r}-\left(\frac{\partial^{2} q^{\prime j}}{\partial q^{k} \partial q^{l}} \frac{\partial q^{i}}{\partial q^{\prime j}} q_{\mu}^{k} q_{v}^{l}+\frac{\partial^{2} q^{j}}{\partial q^{k} \partial x^{\mu}} \frac{\partial q^{i}}{\partial q^{\prime j}} q_{v}^{k}+\frac{\partial^{2} q^{\prime j}}{\partial q^{k} \partial x^{v}} \frac{\partial q^{i}}{\partial q^{\prime j}} q_{\mu}^{k}-\frac{\partial^{2} x^{\prime \gamma}}{\partial x^{\mu} \partial x^{v}} \frac{\partial x^{\rho}}{\partial x^{\prime \gamma}} q_{\rho}^{i}\right) p_{i}^{\mu ; v} \\
& -\left(\frac{\partial^{2} q^{\prime j}}{\partial x^{\mu} \partial x^{v}} \frac{\partial q^{i}}{\partial q^{\prime j}}-\frac{\partial^{2} x^{\prime \gamma}}{\partial x^{\mu} \partial x^{v}} \frac{\partial x^{\rho}}{\partial x^{\prime \gamma}} \frac{\partial q^{\prime j}}{\partial x^{\rho}} \frac{\partial q^{i}}{\partial q^{\prime j}}\right) p_{i}^{\mu ; v}
\end{aligned}
$$

enquanto que a lei de transformação para as coordenadas induzidas de $\bar{J}^{2 \circledast} E$ será:

$$
\begin{aligned}
p_{j}^{\prime \alpha ; \beta} & =\operatorname{det}\left(\frac{\partial x}{\partial x^{\prime}}\right) \frac{\partial q^{i}}{\partial q^{\prime j}} \frac{\partial x^{\prime \alpha}}{\partial x^{\mu}} \frac{\partial x^{\prime \beta}}{\partial x^{v}} p_{i}^{\mu ; v} \\
\bar{r}^{\prime} & =\operatorname{det}\left(\frac{\partial x}{\partial x^{\prime}}\right)\left(\bar{r}-\left(\frac{\partial^{2} q^{j}}{\partial q^{k} \partial q^{l}} \frac{\partial q^{i}}{\partial q^{\prime j}} q_{\mu}^{k} q_{v}^{l}+\frac{\partial^{2} q^{j}}{\partial q^{k} \partial x^{\mu}} \frac{\partial q^{i}}{\partial q^{\prime j}} q_{v}^{k}+\frac{\partial^{2} q^{j}}{\partial q^{k} \partial x^{v}} \frac{\partial q^{i}}{\partial q^{\prime j}} q_{\mu}^{k}-\frac{\partial^{2} x^{\prime \gamma}}{\partial x^{\mu} \partial x^{v}} \frac{\partial x^{\rho}}{\partial x^{\prime \gamma}} q_{\rho}^{i}\right) p_{i}^{\mu ; v}\right) \\
& -\operatorname{det}\left(\frac{\partial x}{\partial x^{\prime}}\right)\left(\frac{\partial^{2} q^{\prime j}}{\partial x^{\mu} \partial x^{v}} \frac{\partial q^{i}}{\partial q^{\prime j}}-\frac{\partial^{2} x^{\prime \gamma}}{\partial x^{\mu} \partial x^{v}} \frac{\partial x^{\rho}}{\partial x^{\prime \gamma}} \frac{\partial q^{\prime j}}{\partial x^{\rho}} \frac{\partial q^{i}}{\partial q^{\prime j}}\right) p_{i}^{\mu ; \nu} .
\end{aligned}
$$


14

1 | FIBRADOS DE JATOS E COJATOS

Por outro lado, a lei de transformação para as coordenadas induzidas de $J^{2 \star} E$ será a seguinte:

$$
\begin{aligned}
p_{j}^{\prime \alpha \beta} & =\frac{\partial q^{i}}{\partial q^{\prime j}} \frac{\partial x^{\prime \alpha}}{\partial x^{\mu}} \frac{\partial x^{\prime \beta}}{\partial x^{v}} p_{i}^{\mu v} \\
r^{\prime} & =r-\left(\frac{\partial^{2} q^{\prime j}}{\partial q^{k} \partial q^{l}} \frac{\partial q^{i}}{\partial q^{\prime j}} q_{\mu}^{k} q_{v}^{l}+\frac{\partial^{2} q^{j}}{\partial q^{k} \partial x^{\mu}} \frac{\partial q^{i}}{\partial q^{\prime j}} q_{v}^{k}+\frac{\partial^{2} q^{j}}{\partial q^{k} \partial x^{v}} \frac{\partial q^{i}}{\partial q^{\prime j}} q_{\mu}^{k}-\frac{\partial^{2} x^{\prime \gamma}}{\partial x^{\mu} \partial x^{v}} \frac{\partial x^{\rho}}{\partial x^{\prime \gamma}} q_{\rho}^{i}\right) p_{i}^{\mu v} \\
& -\left(\frac{\partial^{2} q^{\prime j}}{\partial x^{\mu} \partial x^{v}} \frac{\partial q^{i}}{\partial q^{\prime j}}-\frac{\partial^{2} x^{\prime \gamma}}{\partial x^{\mu} \partial x^{v}} \frac{\partial x^{\rho}}{\partial x^{\prime \gamma}} \frac{\partial q^{\prime j}}{\partial x^{\rho}} \frac{\partial q^{i}}{\partial q^{\prime j}}\right) p_{i}^{\mu v}
\end{aligned}
$$

enquanto que a lei de transformação para as coordenadas induzidas de $J^{2 \star} E$ será:

$$
\begin{aligned}
p_{j}^{\prime \alpha \beta} & =\operatorname{det}\left(\frac{\partial x}{\partial x^{\prime}}\right) \frac{\partial q^{i}}{\partial q^{\prime j}} \frac{\partial x^{\prime \alpha}}{\partial x^{\mu}} \frac{\partial x^{\prime \beta}}{\partial x^{v}} p_{i}^{\mu \nu} \\
r^{\prime} & =\operatorname{det}\left(\frac{\partial x}{\partial x^{\prime}}\right)\left(r-\left(\frac{\partial^{2} q^{j}}{\partial q^{k} \partial q^{l}} \frac{\partial q^{i}}{\partial q^{\prime j}} q_{\mu}^{k} q_{v}^{l}+\frac{\partial^{2} q^{j}}{\partial q^{k} \partial x^{\mu}} \frac{\partial q^{i}}{\partial q^{\prime j}} q_{v}^{k}+\frac{\partial^{2} q^{j}}{\partial q^{k} \partial x^{v}} \frac{\partial q^{i}}{\partial q^{\prime j}} q_{\mu}^{k}-\frac{\partial^{2} x^{\prime \gamma}}{\partial x^{\mu} \partial x^{v}} \frac{\partial x^{\rho}}{\partial x^{\prime \gamma}} q_{\rho}^{i}\right) p_{i}^{\mu \nu}\right) \\
& -\operatorname{det}\left(\frac{\partial x}{\partial x^{\prime}}\right)\left(\frac{\partial^{2} q^{\prime j}}{\partial x^{\mu} \partial x^{v}} \frac{\partial q^{i}}{\partial q^{\prime j}}-\frac{\partial^{2} x^{\prime \gamma}}{\partial x^{\mu} \partial x^{v}} \frac{\partial x^{\rho}}{\partial x^{\prime \gamma}} \frac{\partial q^{\prime j}}{\partial x^{\rho}} \frac{\partial q^{i}}{\partial q^{\prime j}}\right) p_{i}^{\mu v} .
\end{aligned}
$$




\section{Capítulo 2}

\section{Estruturas Simpléticas Generalizadas}

\subsection{Estruturas Simpléticas}

Antes de iniciarmos o assunto principal, precisamos definir o conceito de estrutura simplética e suas generalizações (multissimplética e polissimplética) sobre uma dada variedade.

Seja $W$ um espaço vetorial e $\bigwedge^{k} W^{*}$ o espaço de $k$-formas sobre $W$. Denotamos a contração de uma forma $\alpha \in \bigwedge^{k} W^{*}$ com um vetor $w \in W$ por $i_{w} \alpha \in \bigwedge^{k-1} W^{*}$. Suponha que o par $(W, \omega)$ seja tal que $\omega \in \bigwedge^{2} W^{*}$. Dizemos que $(W, \omega)$ é um espaço simplético se o mapa contração $\omega^{b}: W \rightarrow W^{*}, w \mapsto i_{w} \omega$, for bijetor, i.e, se $\omega$ for uma 2 -forma não-degenerada. Estendendo essa definição para variedades, dizemos que uma variedade $P$ munida de uma 2-forma $\omega \in \Omega^{2}(P)$ fechada é simplética se seu espaço tangente $\left(T_{p} P, \omega_{p}\right)$ for simplético para todo $p \in P$; note que isso força a dimensão de $P \operatorname{ser} \operatorname{par}(\operatorname{dim} P=2 n)$. Se $\omega$ for uma forma fechada degenerada com ker $\omega$ de posto constante, i.e, se $\omega^{b}$ possuir núcleo não-trivial de dimensão constante, dizemos que a variedade $P$ é pré-simplética.

Teorema de Darboux: Sejam $P$ uma $2 n$-variedade e $\omega \in \Omega^{2}(P)$. Então $\omega$ é uma forma simplética se, e somente se, em torno de cada ponto $p \in P$, existe um sistema de coordenadas locais $x^{i}$ tal que

$$
\omega=\frac{1}{2} \omega_{i j} d x^{i} \wedge d x^{j}
$$

com funções $\omega_{i j}$ constantes.

Este é um dos teoremas mais importantes da geometria simplética e daremos a ideia de sua demonstração.

Tratando-se de um teorema de natureza local, basta prová-lo para vizinhanças abertas de 0 em $\mathbb{R}^{2 n}$, escrevendo $\omega=\frac{1}{2} \omega_{i j} d x^{i} \wedge d x^{j}$ com funções $\omega_{i j}$ e introduzindo a forma $\omega_{1}=\frac{1}{2} \omega_{i j}^{(0)} d x^{i} \wedge d x^{j}$ com coeficientes constantes. Então, a afirmação de que existe um novo sistema de coordenadas locais $\left(y^{k}\right)$ em que $\omega$ assume a forma $\frac{1}{2} \omega_{k l}^{(0)} d y^{k} \wedge d y^{l}$ é equivalente à afirmação de que existe 
um difeomorfismo local $F_{1}$ tal que $\omega=F_{1}^{*} \omega_{1}$, pois, definindo $F_{1}$ por $y=F_{1}(x)$, ambas as condições equivalem a

$$
\omega_{i j}=\omega_{k l}^{(0)} \frac{\partial y^{k}}{\partial x^{i}} \frac{\partial y^{l}}{\partial x^{j}} .
$$

Tal difeomorfismo $F_{1}$ é construído da seguinte forma.

Defina a 2 -forma $\tilde{\omega}=\omega_{1}-\omega$ e uma família parametrizada de 2 -formas $\omega_{t}=\omega+t \tilde{\omega}$, que são também ambas fechadas e não-degeneradas. Pelo lema de Poincaré, existem uma 1-forma $\alpha$ tal que $\tilde{\omega}=d \alpha$ e $\alpha(0)=0$ e uma família parametrizada de campos vetoriais $X_{t}$ construída de forma que $i_{X_{t}} \omega_{t}=-\alpha$.

Seja $F_{t}$ o fluxo local de $X_{t}$. Então temos que $\frac{d}{d t}\left(F_{t}^{*} \omega_{t}\right)=0$ e $F_{1}^{*} \omega_{1}=F_{0}^{*} \omega_{0}=\omega$. ([4])

O exemplo padrão de uma variedade simplética é o de um fibrado cotangente: se $P=T^{*} Q$ onde $Q$ é uma variedade qualquer, então $P$ vem munido de uma estrutura simplética natural onde $\omega=-d \theta$ e $\theta$ é uma 1-forma chamada de forma canônica, definida por

$$
\theta_{\alpha}(v)=\alpha\left(T_{\alpha} \tau_{Q}^{*} \cdot v\right)
$$

para $\alpha \in T^{*} Q, v \in T_{\alpha}\left(T^{*} Q\right)$, onde $\tau_{Q}^{*}: T^{*} Q \rightarrow Q$ é a projeção canônica. Em coordenadas canônicas, i.e., coordenadas locais $\left(q^{i}, p_{i}\right)$ de $P$ induzidas por coordenadas locais $q^{i}$ de $Q, \theta$ assume a forma

$$
\theta=p_{i} d q^{i}
$$

de modo que

$$
\omega=d q^{i} \wedge d p_{i}
$$

Podem-se ampliar estas construções a fibrados sobre uma variedade qualquer, o que leva à definição de estruturas multissimpléticas e polissimpléticas, como veremos a seguir. Assim como as estruturas simpléticas, essas possuem um aspecto puramente algébrico, e outro geométrico. Em ambos os casos, começamos pelo primeiro.

\subsection{Estruturas Multissimpléticas}

Considere um espaço vetorial $Y$ (de dimensão finita) junto com um subespaço vetorial $V$ e o correspondente espaço quociente $Z \cong Y / V$, que formam uma sequência exata:

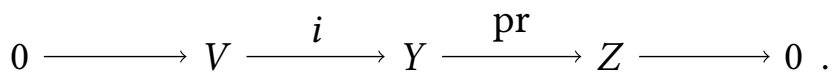

Chamando os vetores de $V$ de vetores verticais, diremos que uma $k$-forma $\alpha$ em $\bigwedge^{k} Y^{*}$ é $(k-r)$-horizontal se sua contração com $r+1$ vetores de $V$ é nula, ou seja,

$$
i_{v_{1}} \ldots i_{v_{r+1}} \alpha=0 \text { para } v_{1}, \ldots, v_{r+1} \in V
$$

O espaço de $k$-formas $(k-r)$-horizontais sobre $Y$ é denotada por $\bigwedge_{r}^{k} Y^{*}$. Note que, dado $\alpha \in \bigwedge_{r}^{k} Y^{*}$, as contrações de $\alpha$ definem o mapa linear $\alpha^{b}: Y \rightarrow \bigwedge_{r}^{k-1} Y^{*}$. Em particular, note que $\alpha^{b}: V \rightarrow \bigwedge_{r-1}^{k-1} Y^{*}$. 
Defina o aniquilador de um subespaço $L$ de $Y$ como o subespaço $L^{\perp}$ de $Y^{*}$ das 1-formas que se anulam sobre $L$. Note que, através da sequência exata acima, $V^{\perp}$ pode ser identificado com o espaço dual $Z^{*}$ do espaço quociente $Y / V$ e que se $L \subset V$, então $V^{\perp} \subset L^{\perp}$; neste caso, defina $\bigwedge_{r-1}^{k} L^{\perp}=\bigwedge^{k} L^{\perp} \cap \bigwedge_{r-1}^{k} Y^{*}$.

Dizemos que uma $(k+1)$-forma $(k+1-r)$-horizontal $\omega \in \bigwedge_{r}^{k+1} Y^{*}$ é multissimplética de posto $N$ se $\omega$ for uma forma não-degenerada (ker $\omega^{b}=\{0\}$ ) e admitir um subespaço $L$ de $V$, de codimensão $N$, tal que $\omega^{b}(L)=\bigwedge_{r-1}^{k} L^{\perp}$. Tal subespaço $L$ é chamado de espaço multilagrangiano, e $Y$ é dito espaço multissimplético. Supondo que $\operatorname{dim} Z=n$, o caso de interesse físico ocorre com $k=n$ e $r=2$; no caso da mecânica, $k=n=1$ e a condição de horizontalidade é automaticamente satisfeita. A grande novidade é que, quando $n>1$, o subespaço multilagrangiano é únicamente determinado por $\omega$.

Essas construções se estendem fibra a fibra para fibrados: dado um fibrado $(E, \pi, M)$, substituímos $Z$ pelo fibrado tangente à base $M, Y$ pelo fibrado tangente ao espaço total $E$ e $V$ pelo fibrado vertical. Sejam $(P, \rho, M)$ um fibrado qualquer e $\omega$ uma $(k+1)$-forma $(k+1-r)$-horizontal, $\omega \in \Gamma\left(\bigwedge_{r}^{k+1} T^{*} P\right)$. Dizemos que $\omega$ é multissimplética de posto $N$ se $\omega$ for fechada $(d \omega=0)$ e tal que, para cada $p \in P, \omega_{p}$ seja uma forma multissimplética de posto $N$ com respeito à sequência exata

$$
0 \longrightarrow V_{p} P \stackrel{i}{\longrightarrow} T_{p} P \stackrel{T_{p} \rho}{\longrightarrow} T_{x} M \longrightarrow 0 .
$$

Assim sendo, a distribuição $L$ sobre $P$ é de posto constante e chamada de distribuição multilagrangiana, e $P$ é dito fibrado multissimplético. Se $\omega$ for uma forma fechada degenerada com núcleo de dimensão constante, dizemos que o fibrado $P$ é pré-multissimplético.

Teorema de Darboux: Seja $P$ um fibrado pré-multissimplético sobre uma $n$-variedade $M$ com forma pré-multissimplética $\omega \in \Gamma\left(\bigwedge_{r}^{k+1} T^{*} P\right)$ de posto $N$, com $1 \leqslant r \leqslant k$ e $k+1-r \leqslant n$. Suponha ainda que a distribuição pré-multilagrangiana $L$ seja involutiva (casos excepcionais p.29-30 [8]). Então, em torno de qualquer ponto em $P$, existe um sistema de coordenadas locais $\left(x^{\mu}, q^{i}, p_{i_{1} \ldots i_{s} ; \mu_{1} \ldots \mu_{k-s}}, r^{\kappa}\right)(\operatorname{com} 0 \leqslant s \leqslant r-1,1 \leqslant \mu \leqslant n, 1 \leqslant i \leqslant N$, $\left.1 \leqslant i_{1}<\cdots<i_{s} \leqslant N, 1 \leqslant \mu_{1}<\cdots<\mu_{k-s} \leqslant N, 1 \leqslant \kappa \leqslant \operatorname{dim} \operatorname{ker} \omega\right)$ tal que

$$
\omega=-\sum_{s=0}^{r-1} \frac{1}{s !} \frac{1}{(k-s) !} d p_{i_{1} \ldots i_{s} ; \mu_{1} \ldots \mu_{k-s}} \wedge d q^{i_{1}} \wedge \ldots \wedge d q^{i_{s}} \wedge d x^{\mu_{1}} \wedge \ldots \wedge d x^{\mu_{k-s}}
$$

No caso de interesse físico, $\operatorname{com} k=n$ e $r=2$, temos que

$$
\omega=d q^{i} \wedge d p_{i}^{\mu} \wedge d^{n} x_{\mu}-d p \wedge d^{n} x .
$$

O exemplo padrão de um fibrado multissimplético é o de um fibrado de formas horizontais: se $P=\bigwedge_{r-1}^{k} T^{*} E$ onde $E$ é o espaço total de um fibrado $(E, \pi, M)$ qualquer, então $P$ vem munido de uma estrutura multissimplética natural onde $\omega=-d \theta$ e $\theta$ é uma $k$-forma chamada de forma multicanônica, ou simplesmente forma canônica, definida por

$$
\theta_{\alpha}\left(v_{1}, \ldots, v_{k}\right)=\alpha\left(T_{\alpha} \pi_{r-1}^{k} \cdot v_{1}, \ldots, T_{\alpha} \pi_{r-1}^{k} \cdot v_{k}\right)
$$

para $\alpha \in \bigwedge_{r-1}^{k} T^{*} E, v_{1}, \ldots, v_{k} \in T_{\alpha}\left(\bigwedge_{r-1}^{k} T^{*} E\right)$, onde $\pi_{r-1}^{k}: \bigwedge_{r-1}^{k} T^{\star} E \rightarrow E$ é a projeção 
canônica. Em coordenadas locais adaptadas, i.e, coordenadas locais $\left(x^{\mu}, q^{i}, p_{i_{1} \ldots i_{s} ; \mu_{1} \ldots \mu_{k-s}}\right)$ de $P$ induzidas por coordenadas locais $\left(x^{\mu}, q^{i}\right)$ de $E, \theta$ assume a forma

$$
\theta=\sum_{s=0}^{r-1} \frac{1}{s !} \frac{1}{(k-s) !} p_{i_{1} \ldots i_{s} ; \mu_{1} \ldots \mu_{k-s}} \wedge d q^{i_{1}} \wedge \ldots \wedge d q^{i_{s}} \wedge d x^{\mu_{1}} \wedge \ldots \wedge d x^{\mu_{k-s}}
$$

No caso de interesse físico, com $k=n$ e $r=2$, temos que $\bigwedge_{1}^{n} T^{\star} E \cong J^{\circledast} E$ e

$$
\theta=p_{i}^{\mu} d q^{i} \wedge d^{n} x_{\mu}+p d^{n} x
$$

\subsection{Estruturas Polissimpléticas}

Sejam $V$ e $\hat{T}$ dois espaços vetoriais de dimensões finitas e $\hat{\omega} \in \bigwedge^{k+1} V^{*} \otimes \hat{T}$ uma $(k+1)$-forma sobre $V$ a valores em $\hat{T}$. Novamente, definimos o mapa contração $\hat{\omega}^{b}: V \rightarrow \bigwedge^{k} V^{*} \otimes \hat{T}$ por $\hat{\omega}^{b}(v)=i_{v} \hat{\omega}$.

Como antes, dado um subespaço $L \subset V$, denotamos o seu aniquilador por $L^{\perp} \subset V^{*}$. Dizemos que $\hat{\omega}$ é polissimplética de posto $N$ se $\hat{\omega}$ for não-degenerada (ker $\hat{\omega}^{b}=\{0\}$ ) e admitir um subespaço $L$ de $V$, de codimensão $N$, tal que $\hat{\omega}^{b}(L)=\left(\bigwedge^{k} L^{\perp}\right) \otimes \hat{T}$. Tal subespaço $L$ é chamado de espaço polilagrangiano e $V$ é dito espaço polissimplético. O caso de interesse físico ocorre com $k=1$.

Assim como a estrutura multissimplética, a estrutura polissimplética também possui compatibilidade com fibrados. Sejam $(P, \rho, M)$ um dado fibrado qualquer e $(\hat{T}, \tau, M)$ um fibrado vetorial. Considere o fibrado $\bigwedge^{k} V^{*} P$ das $k$-formas verticais sobre $P$ e o fibrado $\bigwedge^{k} V^{*} P \otimes \rho^{*} \hat{T}$ das $k$-formas verticais a valores em $\hat{T}$ (seria mais preciso dizer a valores em $\rho^{*} \hat{T}$ ). Uma seção de $\bigwedge^{k} V^{*} P \otimes \rho^{*} \hat{T}$ é chamada de $k$-forma (diferencial) vertical a valores em $\hat{T}$; o espaço de todas essas formas é denotado por

$$
\Omega_{V}^{k}\left(P ; \rho^{*} \hat{T}\right)=\Gamma\left(\bigwedge^{k} V^{*} P \otimes \rho^{*} \hat{T}\right) .
$$

É importante notar que $\Omega_{V}^{k}\left(P ; \rho^{*} \hat{T}\right)$ não é um subespaço de $\Omega^{k}\left(P ; \rho^{*} \hat{T}\right)$, sendo na verdade o quociente

$$
\Omega_{V}^{k}\left(P ; \rho^{*} \hat{T}\right)=\Omega^{k}\left(P ; \rho^{*} \hat{T}\right) / \Omega_{k}^{k}\left(P ; \rho^{*} \hat{T}\right) .
$$

Assim, um elemento de $\Omega_{V}^{k}\left(P ; \rho^{*} \hat{T}\right)$ expressa uma classe de equivalência de $k$-formas sobre $P$ a valores em $\hat{T}$.

Essa construção de formas diferenciais verticais sobre o espaço total $P$ de um fibrado torna possível aplicarmos uma variação do cálculo de Cartan usual, na qual campos vetoriais dão lugar a campos vetoriais verticais e formas diferenciais dão espaço a formas diferenciais verticais. Essa variação funciona não apenas para formas a valores escalares ou formas a valores num espaço vetorial fixo, mas também para formas a valores em um fibrado vetorial sobre $P$, desde que esse seja o pull-back de um fibrado vetorial sobre $M$, e ela ocorre de tal modo que todas as operações do cálculo usual continuam bem definidas e satisfazendo as regras do cálculo de Cartan padrão. [8] 
Para o uso desejado da estrutura polissimplética nesse trabalho, precisaremos apenas da derivada exterior vertical

$$
\begin{array}{ccc}
d_{V}: \Omega_{V}^{k}\left(P ; \rho^{*} \hat{T}\right) & \longrightarrow & \Omega_{V}^{k+1}\left(P ; \rho^{*} \hat{T}\right) \\
\alpha & \longmapsto & d_{V} \alpha
\end{array}
$$

definida por

$$
\begin{aligned}
d_{V} \alpha\left(X_{0}, \ldots, X_{k}\right)= & \sum_{i=0}^{k}(-1)^{i} X_{i} \cdot\left(\alpha\left(X_{0}, \ldots, \widehat{X}_{i}, \ldots, X_{k}\right)\right) \\
& +\sum_{0 \leqslant i<j \leqslant k}(-1)^{i+j} \alpha\left(\left[X_{i}, X_{j}\right], X_{0}, \ldots, \widehat{X}_{i}, \ldots, \widehat{X}_{j}, \ldots, X_{k}\right),
\end{aligned}
$$

onde $X_{0}, \ldots, X_{k} \in \mathfrak{X}_{V}(P)=\Gamma(V P)$.

Nessas mesmas condições, dizemos que uma $(k+1)$-forma vertical a valores em $\hat{T}$, $\hat{\omega} \in \Omega_{V}^{k+1}\left(P ; \rho^{*} \hat{T}\right)$, é polissimplética de posto $N$ se $\hat{\omega}$ for fechada $\left(d_{V} \hat{\omega}=0\right)$ e, para cada ponto $p \in P, \hat{\omega}_{p}$ for uma forma polissimplética de posto $N$ sobre o espaço vertical $V_{p} P$. Assim sendo, a distribuição $L$ sobre $P$ é de posto constante e chamada de distribuição polilagrangiana, e $P$ é dito fibrado polissimplético. Se $\hat{\omega}$ for uma forma fechada degenerada com núcleo de dimensão constante, dizemos que o fibrado $P$ é pré-polissimplético.

Teorema de Darboux: Seja $P$ um fibrado pré-polissimplético sobre uma $n$-variedade $M$ com forma pré-polissimplética $\hat{\omega} \in \Omega_{V}^{k+1}\left(P ; \rho^{*} \hat{T}\right)$ de posto $N$ a valores em um fibrado vetorial $\hat{T}$ de dimensão $\hat{n}$ sobre a variedade $M$. Suponha ainda que a distribuição prépolilagrangiana $L$ seja involutiva e que $\left\{\hat{e}_{a} \mid 1 \leqslant a \leqslant \hat{n}\right\}$ seja uma base de seções locais de $\hat{T}$ (casos excepcionais p.29-30 [8]). Então, em torno de qualquer ponto em $P$, existe um sistema de coordenadas locais $\left(x^{\mu}, q^{i}, p_{i_{1} \ldots i_{k}}^{a}, r^{\kappa}\right)(\operatorname{com} 1 \leqslant \mu \leqslant n, 1 \leqslant a \leqslant \hat{n}, 1 \leqslant i \leqslant N$, $\left.1 \leqslant i_{1}<\cdots<i_{k} \leqslant N, 1 \leqslant \kappa \leqslant \operatorname{dim} \operatorname{ker} \hat{\omega}\right)$ tal que

$$
\hat{\omega}=-\frac{1}{k !} d p_{i_{1} \ldots i_{k}}^{a} \wedge d q^{i_{1}} \wedge \ldots \wedge d q^{i_{k}} \otimes \hat{e}_{a} .
$$

No caso de interesse físico, $\operatorname{com} k=1$, temos que

$$
\hat{\omega}=d q^{i} \wedge d p_{i}^{a} \otimes \hat{e}_{a}
$$

O exemplo padrão de um fibrado polissimplético é o de um fibrado de formas verticais: se $P=\bigwedge^{k} V^{*} E \otimes \pi^{*} \hat{T}$ onde $E$ é o espaço total de um fibrado $(E, \pi, M)$ qualquer e $\hat{T}$ é um fibrado vetorial qualquer sobre $M$, então $P$ vem munido de uma estrutura polissimplética natural onde $\hat{\omega}=-d_{V} \hat{\theta}$ e $\hat{\theta}$ é uma $k$-forma chamada de forma policanônica, ou simplesmente forma canônica, definida por

$$
\hat{\theta}_{\alpha}\left(v_{1}, \ldots, v_{k}\right)=\alpha\left(T_{\alpha} \pi^{k} \cdot v_{1}, \ldots, T_{\alpha} \pi^{k} \cdot v_{k}\right)
$$

para $\alpha \in \bigwedge^{k} V^{*} E \otimes \pi^{*} \hat{T}, v_{1}, \ldots, v_{k} \in V_{\alpha}\left(\bigwedge^{k} V^{*} E \otimes \pi^{*} \hat{T}\right)$, onde $\pi^{k}: \bigwedge^{k} V^{*} E \otimes \pi^{*} \hat{T} \rightarrow E$ é a projeção canônica. Em coordenadas locais adaptadas, i.e., coordenadas locais $\left(x^{\mu}, q^{i}, p_{i_{1} \ldots i_{k}}^{a}\right)$ 
de $P$ induzidas por coordenadas locais $\left(x^{\mu}, q^{i}\right)$ de $E, \hat{\theta}$ assume a forma

$$
\hat{\theta}=\frac{1}{k !} p_{i_{1} \ldots i_{k}}^{a} d q^{i_{1}} \wedge \ldots \wedge d q^{i_{k}} \otimes \hat{e}_{a} .
$$

No caso de interesse físico, com $k=1$ e $\hat{T}=\bigwedge^{n-1} T^{*} M$, temos que $V^{*} E \otimes \pi^{*} \hat{T} \cong \vec{J}^{\circledast} E$ e

$$
\hat{\theta}=p_{i}^{\mu} d q^{i} \otimes d^{n} x_{\mu}
$$

\subsection{Símbolos}

Toda estrutura pré-multissimplética induz, de forma canônica, uma estrutura prépolissimplética. Seja $\omega$ uma $(k+1)$-forma $(k+1-r)$-horizontal sobre $P$. Dizemos que o símbolo $\hat{\omega}$ de $\omega$ é a $r$-forma vertical sobre $P$ tomando valores em $\hat{T}=\bigwedge^{k+1-r} T^{*} M$ tal que

$$
\hat{\omega}\left(X_{1}, \ldots, X_{r}\right)=i_{X_{1}} \ldots i_{X_{r}} \omega \text {, para } X_{1}, \ldots, X_{r} \in \mathfrak{X}_{V}(P) .
$$

Teorema: Seja $(P, \rho, M)$ um fibrado. Suponha que $\omega$ seja uma forma pré-multissimplética com espaço pré-multilagrangiano $L$, então seu símbolo $\hat{\omega}$ é uma forma pré-polissimplética com espaço pré-polilagrangiano $L$ e $\operatorname{ker} \omega \subset \operatorname{ker} \hat{\omega}$. 


\section{Capítulo 3}

\section{Formalismo Hamiltoniano de Primeira Ordem e Extensões}

\subsection{Estruturas Simpléticas e Multissimpléticas em Primeira Ordem}

\subsubsection{Caso Unidimensional}

A mecânica clássica se propõe a formular equações de movimento para sistemas de partículas que preveem o comportamento do sistema a partir de suas condições iniciais. De maneira geral, essas equações são equações diferenciais, inicialmente de segunda ordem, como na lei de Newton ou no formalismo lagrangiano, enquanto que no formalismo hamiltoniano são reduzidas a sistemas de equações diferenciais de primeira ordem. Suas soluções são curvas parametrizadas pelo tempo absoluto de Newton numa variedade $P_{0}$, chamada espaço de fase, em que, para um sistema com $N$ graus de liberdade, as coordenadas generalizadas $\left(q^{i}\right)_{i=1, \ldots, N}$ e os momentos generalizados das partículas $\left(p_{i}\right)_{i=1, \ldots, N}$ servem como coordenadas locais. A estrutura básica do espaço de fase é a de uma variedade simplética, em que a forma simplética $\omega_{0}$ nas referidas coordenadas canônicas $\left(q^{i}, p_{i}\right)$ se escreve como

$$
\omega_{0}=d q^{i} \wedge d p_{i} .
$$

O caso padrão ocorre quando o $P_{0}$ é o fibrado cotangente $T^{\star} Q$ de uma variedade $Q$ chamada de espaço de configuração. Neste caso $\omega_{0}$ é exata, $\omega_{0}=-d \theta_{0}$, onde $\theta_{0}$ é chamada forma canônica, que em coordenadas canônicas se escreve como

$$
\theta_{0}=p_{i} d q^{i}
$$

Outro ingrediente básico do formalismo hamiltoniano é a hamiltoniana, que é uma função $H$ a valores reais no espaço de fase $P_{0}$. Então as equações de movimento na sua forma hamiltoniana correspondem a um campo vetorial hamiltoniano $X_{H}$ dado por

$$
i_{X_{H}} \omega_{0}=d H \text {, }
$$


ou, em coordenadas canônicas,

$$
X_{H}=\frac{\partial H}{\partial p_{i}} \frac{\partial}{\partial q^{i}}-\frac{\partial H}{\partial q^{i}} \frac{\partial}{\partial p_{i}} .
$$

De fato, a equação de fluxo para este $X_{H}$ é as equações de Hamilton usuais,

$$
\frac{d p_{i}}{d t}(t)=-\frac{\partial H}{\partial q^{i}}\left(q^{i}(t), p_{i}(t)\right) \quad \frac{d q^{i}}{d t}(t)=\frac{\partial H}{\partial p_{i}}\left(q^{i}(t), p_{i}(t)\right)
$$

No entanto, essa versão do formalismo hamiltoniano é válida apenas para sistemas autônomos. Para incluir sistemas não-autônomos, i.e, para hamiltonianas que dependem explicitamente do tempo, é necessário tomar uma extensão do espaço de fase $\mathbb{R} \times P_{0}$, com coordenadas locais $\left(t, q^{i}, p_{i}\right)$. Entretanto, este "espaço de fase simplesmente estendido" não pode ser uma variedade simplética. Para retornar ao âmbito da geometria simplética é necessário efetuar mais uma extensão e, conforme já sugerido por Élie Cartan, considerar o "espaço de fase duplamente estendido", $P=\mathbb{R} \times P_{0} \times \mathbb{R}$, com coordenadas locais $\left(t, q^{i}, p_{i}, E\right)$. Obviamente, $t$ é uma coordenada tipo tempo e $E$ é uma coordenada tipo energia, e a forma simplética estendida sobre $P$ é, em coordenadas canônicas $\left(t, q^{i}, p_{i}, E\right)$,

$$
\omega=d q^{i} \wedge d p_{i}-d E \wedge d t
$$

e, no caso de $P_{0}$ ser um fibrado cotangente, vale $\omega=-d \theta$, onde

$$
\theta=p_{i} d q^{i}+E d t
$$

Agora, a hamiltoniana $H$ é uma função a valores reais no espaço de fase simplesmente estendido $\mathbb{R} \times P_{0}$, que pode ser considerada como definindo um mapa do espaço de fase simplesmente estendido para o espaço de fase duplamente estendido pondo $E=-H\left(t, q^{i}, p_{i}\right)$, pelo qual introduzimos o pull-back das formas diferenciais acima, i.e,

$$
\omega_{H}=d q^{i} \wedge d p_{i}+d H \wedge d t=d q^{i} \wedge d p_{i}+\frac{\partial H}{\partial q^{i}} d q^{i} \wedge d t+\frac{\partial H}{\partial p_{i}} d p_{i} \wedge d t
$$

e, no caso de $P_{0}$ ser um fibrado cotangente, vale $\omega_{H}=-d \theta_{H}$, onde

$$
\theta_{H}=p_{i} d q^{i}-H d t
$$

Agora, as equações de movimento na sua forma hamiltoniana podem ser expressas na seguinte forma: uma curva $\varphi$ em $P_{0}$ é solução se, e somente se, para qualquer campo vetorial $X$ sobre $\mathbb{R} \times P_{0}$ que é vertical no sentido de que sua componente ao longo de $\frac{\partial}{\partial t}$ é zero, de modo que

$$
X=X^{i} \frac{\partial}{\partial q^{i}}+X_{i} \frac{\partial}{\partial p_{i}}
$$

vale

$$
\varphi^{*} i_{X} \omega_{H}=0
$$


De fato, é fácil ver que essa condição é equivalente às equações de Hamilton usuais,

$$
\frac{d p_{i}}{d t}(t)=-\frac{\partial H}{\partial q^{i}}\left(t, q^{i}(t), p_{i}(t)\right) \quad \frac{d q^{i}}{d t}(t)=\frac{\partial H}{\partial p_{i}}\left(t, q^{i}(t), p_{i}(t)\right) .
$$

\subsubsection{Caso Multidimensional}

O próximo passo que devemos dar com o formalismo é a sua extensão para a teoria de campos, o que requer a substituição da reta $\mathbb{R}$ do tempo absoluto de Newton pela variedade que representa o espaço-tempo e é denotada por $M$, substituindo a coordenada tempo $t$ por coordenadas locais $x^{\mu}$. Nesse contexto, é exigido que tratemos as coordenadas do espaçotempo como variáveis independentes. Outra mudança essencial provém do fato de que em teoria de campos existe uma diferença intrínseca entre posições e momentos, de modo que não há análogo de transformações canônicas que misturam posições com momentos; portanto não haverá análogo de um espaço de fase $P_{0}$ como acima que não seja o fibrado cotangente $T^{*} Q$ de algum espaço de configuração $Q$. Na verdade, o $P_{0}$ acima desaparece totalmente, sobrando apenas suas extensões simples e dupla. Sendo assim, efetuaremos a seguinte mudança de notação: mantendo a notação $P$ para o espaço de fase duplamente estendido, denotaremos a seguir por $P_{0}$ o espaço de fase simplesmente estendido. O leitor poderá verificar que isso não afetará nenhuma das equações da subseção 3.1.1.

Dito isso, qualquer modelo em teoria de campos tem como ponto de partida a escolha de um fibrado $(E, \pi, M)$ sobre o espaço-tempo, cuja fibra típica $Q$ corresponde ao espaço de configuração da mecânica: suas coordenadas locais $q^{i}$ representam as variáveis dinâmicas básicas da teoria. Assim como a evolução do sistema na mecânica é dada por curvas em $Q$, sujeitas a equações diferenciais ordinárias, a evolução do sistema na teoria de campos é dada por seções de $E$, sujeitas a equações diferenciais parciais. Tratar de primeiras derivadas dos campos requer a introdução do fibrado de jatos $J E$ (para derivadas comuns) e do fibrado de jatos lineares $\vec{J} E$ (para derivadas covariantes). Então, o espaço de fase duplamente estendido $P$ é o fibrado dos cojatos afins torcidos $J^{\circledast} E$, enquanto que o espaço de fase simplesmente estendido $P_{0}$ é o fibrado dos cojatos lineares torcidos $\vec{J}^{\circledast} E$.

Como visto nas seções 2.2 e 2.3, o primeiro vem munido de uma forma canônica $\theta$ que, em coordenadas locais adaptadas $\left(x^{\mu}, q^{i}, p_{i}^{\mu}, p\right)$, assume a forma

$$
\theta=p_{i}^{\mu} d q^{i} \wedge d^{n} x_{\mu}+p d^{n} x
$$

e cuja derivada exterior (a menos de sinal) é a forma multissimplética

$$
\omega=d q^{i} \wedge d p_{i}^{\mu} \wedge d^{n} x_{\mu}-d p \wedge d^{n} x .
$$

No contexto físico, as coordenadas $p_{i}^{\mu}$ são os multimomentos do sistema, enquanto que a coordenada $p$ é interpretada como energia do sistema: esta é dada pela hamiltoniana $H$, que é uma seção de $P$ como fibrado afim sobre $P_{0}$; em coordenadas adaptadas, $p=-H\left(x^{\mu}, q^{i}, p_{i}^{\mu}\right)$. Podemos então formar o pull-back das formas diferenciais acima:

$$
\theta_{H}=p_{i}^{\mu} d q^{i} \wedge d^{n} x_{\mu}-H d^{n} x
$$


$\mathrm{e}$

$$
\omega_{H}=d q^{i} \wedge d p_{i}^{\mu} \wedge d^{n} x_{\mu}+d H \wedge d^{n} x .
$$

Além disso, uma seção $\varphi$ de $P_{0}$ como fibrado sobre $M$ é solução das equações de movimento se, e somente se, para qualquer campo vetorial $X$ sobre $P_{0}$ que é vertical em relação à projeção de $P_{0}$ sobre $M$, de modo que

$$
X=X^{i} \frac{\partial}{\partial q^{i}}+X_{i}^{\mu} \frac{\partial}{\partial p_{i}^{\mu}},
$$

vale

$$
\varphi^{*} i_{X} \omega_{H}=0 .
$$

De fato, é fácil ver que essa condição é equivalente às equações de De Donder-Weyl,

$$
\frac{\partial p_{i}^{\mu}}{\partial x^{\mu}}(x)=-\frac{\partial H}{\partial q^{i}}(\varphi(x)) \quad \frac{\partial q^{i}}{\partial x^{\mu}}(x)=\frac{\partial H}{\partial p_{i}^{\mu}}(\varphi(x)) .
$$

\subsection{Sobre a Extensão à Segunda Ordem}

\subsubsection{Caso Unidimensional}

O próximo passo requer introduzir o fibrado tangente de segunda ordem $T^{2} Q$ que acomoda a segunda derivada de curvas no espaço de configuração $Q$. Para isso, tomaremos inicialmente o fibrado tangente iterado de segunda ordem $T(T Q)$. Qualquer sistema de coordenadas locais $q^{i}$ de $Q$ induz coordenadas locais $\left(q^{i}, \dot{q}^{i}\right)$ de $T Q$ e $\left(q^{i}, \dot{q}_{\mathrm{I}}^{i}, \dot{q}_{\mathrm{II}}^{i}, \ddot{q}^{i}\right)$ de $T(T Q)$, e se passarmos para um novo sistema de coordenadas locais $q^{\prime j}$ de $Q$, teremos a seguinte lei de transformação para as coordenadas locais induzidas:

$$
\dot{q}_{\mathrm{I}}^{\prime j}=\frac{\partial q^{j}}{\partial q^{i}} \dot{q}_{\mathrm{I}}^{i} \quad \dot{q}_{\mathrm{II}}^{\prime j}=\frac{\partial q^{j}}{\partial q^{i}} \dot{q}_{\mathrm{II}}^{i} \quad \ddot{q}^{\prime j}=\frac{\partial q^{j}}{\partial q^{i}} \ddot{q}^{i}+\frac{\partial^{2} q^{j}}{\partial q^{k} \partial q^{l}} \dot{q}_{\mathrm{I}}^{k} \dot{q}_{\mathrm{II}}^{l}
$$

Note que $T(T Q)$ admite duas projeções sobre $T Q$, a saber, $\tau_{T Q}$ e $T \tau_{Q}$; em coordenadas, essas projeções levam $\left(q^{i}, \dot{q}_{\mathrm{I}}^{i}, \dot{q}_{\mathrm{II}}^{i}, \ddot{q}^{i}\right)$ para $\left(q^{i}, \dot{q}_{\mathrm{I}}^{i}\right)$ e para $\left(q^{i}, \dot{q}_{\mathrm{II}}^{i}\right)$, respectivamente, sendo que $T(T Q)$ é um fibrado vetorial sobre $T Q$ em relação a cada uma delas. Define-se o fibrado tangente de segunda ordem de $Q, T^{2} Q$, como o subfibrado de $T(T Q)$ onde essas duas projeções coincidem,

$$
T^{2} Q=\left\{w \in T(T Q) \mid \tau_{T Q} w=T \tau_{Q} w\right\}
$$

O espaço total $T^{2} Q$ assume coordenadas locais $\left(q^{i}, \dot{q}^{i}, \ddot{q}^{i}\right)$, sendo um fibrado afim sobre $T Q$, e seu fibrado vetorial de diferenças $\vec{T}^{2} Q$ sobre $T Q$ assume coordenadas locais $\left(q^{i}, \dot{q}^{i}, \overrightarrow{\ddot{q}}^{i}\right)$. Se passarmos novamente para um novo sistema de coordenadas locais $q^{j}$ de $Q$, teremos a seguinte lei de transformação para as coordenadas locais induzidas:

$$
\dot{q}^{\prime j}=\frac{\partial q^{\prime j}}{\partial q^{i}} \dot{q}^{i} \quad \ddot{q}^{\prime j}=\frac{\partial q^{j}}{\partial q^{i}} \ddot{q}^{i}+\frac{\partial^{2} q^{\prime j}}{\partial q^{k} \partial q^{l}} \dot{q}^{k} \dot{q}^{l} \quad \overrightarrow{\ddot{q}}^{j}=\frac{\partial q^{\prime j}}{\partial q^{i}} \overrightarrow{\ddot{q}}^{i} .
$$



$T_{u}^{2} Q$

Passando a duais, fixe um elemento $u$ de $T Q$ e considere o dual afim do espaço afim

$$
T_{u}^{2 \star} Q=\left\{z: T_{u}^{2} Q \rightarrow \mathbb{R} \mid z \text { é afim }\right\},
$$

assim como o dual espaço linear do espaço vetorial $\vec{T}_{u}^{2} Q$, i.e,

$$
\vec{T}_{u}^{2 *} Q=\left\{\vec{z}: \vec{T}_{u}^{2} Q \rightarrow \mathbb{R} \mid \vec{z} \text { é linear }\right\}
$$

Tomando as uniões disjuntas enquanto $u$ percorre $T Q$, obtemos os fibrados vetoriais $T^{2 \star} Q$ e $\vec{T}^{2 \star} Q$ sobre $T Q$. As coordenadas locais $T^{2 \star} Q$ e $\vec{T}^{2 \star} Q$ induzidas por coordenadas locais $q^{i}$ de $Q$ serão denotadas por $\left(q^{i}, \dot{q}^{i}, r_{i}, r\right)$ e $\left(q^{i}, \dot{q}^{i}, r_{i}\right)$. Além disso, $T^{2 \star} Q$ é um fibrado afim sobre $\vec{T}^{2 *} Q$ com projeção dada pela omissão da coordenada escalar $r$. Os elementos desses espaços são definidos através do pareamento com os elementos de $T^{2} Q$ e de $\vec{T}^{2} Q$ respectivamente de forma que, dados $w \in T^{2} Q$ e $\vec{w} \in \vec{T}^{2} Q$,

$$
\langle z, w\rangle=r_{i} \ddot{q}^{i}+r \quad\langle\vec{z}, \vec{w}\rangle=r_{i} \vec{q}^{i}
$$

Isso implica que, se passarmos para um novo sistema de coordenadas locais $q^{j}$ de $Q$, devemos ter

$$
r_{i} \ddot{q}^{i}+r=r_{j}^{\prime} \ddot{q}^{j}+r^{\prime} \quad r_{i} \overrightarrow{\ddot{q}}^{i}=r_{j}^{\prime} \overrightarrow{\ddot{q}}^{j}
$$

e, portanto, a lei de transformação para as coordenadas induzidas de $T^{2 \star} Q$ e $\vec{T}^{2 \star} Q$ será a seguinte:

$$
r_{j}^{\prime}=\frac{\partial q^{i}}{\partial q^{\prime j}} r_{i} \quad r^{\prime}=r-\frac{\partial q^{i}}{\partial q^{\prime j}} \frac{\partial^{2} q^{j}}{\partial q^{k} \partial q^{l}} \dot{q}^{k} \dot{q}^{l} r_{i}
$$

Nota-se que esses espaços duais $T^{2 \star} Q$ e $\vec{T}^{2 \star} Q$ não possuem estrutura simplética óbvia. Sendo assim, nossa atenção se volta para o fibrado dual $T^{*}(T Q)$ do fibrado tangente iterado $T(T Q)$, de coordenadas induzidas $\left(q^{i}, \dot{q}_{\mathrm{I}}^{i}, p_{i}, r_{i}\right)$. Os elementos de $T^{*}(T Q)$ são definidos através do pareamento com os elementos de $T(T Q)$ de forma que obtemos

$$
r_{i} \ddot{q}^{i}+p_{i} \dot{q}_{\mathrm{II}}^{i}
$$

Se passarmos para um novo sistema de coordenadas locais $q^{\prime j}$, devemos ter

$$
r_{i} \ddot{q}^{i}+p_{i} \dot{q}_{\mathrm{II}}^{i}=r_{j}^{\prime} \ddot{q}^{\prime j}+p_{j}^{\prime} \dot{q}_{\mathrm{II}}^{\prime j}
$$

e portanto a lei de transformação para as coordenadas induzidas de $T^{*}(T Q)$ será

$$
r_{j}^{\prime}=\frac{\partial q^{i}}{\partial q^{\prime j}} r_{i} \quad p_{j}^{\prime}=\frac{\partial q^{i}}{\partial q^{\prime j}} p_{i}-\frac{\partial q^{i}}{\partial q^{\prime m}} \frac{\partial^{2} q^{\prime m}}{\partial q^{k} \partial q^{l}} \frac{\partial q^{k}}{\partial q^{\prime j}} \dot{q}^{l} r_{i} .
$$

Notando que o pull-back $\tau_{Q}^{*}\left(T^{*} Q\right)$ de $T^{*} Q$ para $T Q$ com a projeção $\tau_{Q}: T Q \longrightarrow Q$ pode ser identificado com a soma direta $T Q \oplus T^{*} Q$, podemos concluir destas leis de transformação que os seguintes mapas são bem definidos e são morfismos de fibrados vetoriais sobre $T Q$ : 
as inclusões

$$
\begin{array}{ccc}
\overrightarrow{\mathrm{i}}: \quad T Q \oplus T^{*} Q & \longrightarrow & T^{*}(T Q) \\
\left(q^{i}, \dot{q}^{i}, p_{i}\right) & \longmapsto & \left(q^{i}, \dot{q}^{i}, p_{i}, 0\right)
\end{array},
$$

$\mathrm{e}$

$$
\text { i : } \begin{array}{ccc}
\left(T Q \oplus T^{*} Q\right)_{L} & \longrightarrow & T^{*}(T Q) \\
\left(q^{i}, \dot{q}^{i}, p_{i}\right) & \longmapsto & \left(q^{i}, \dot{q}^{i}, p_{i}, 0\right)
\end{array},
$$

onde $\left(T Q \oplus T^{*} Q\right)_{L}$ é o subfibrado vetorial de codimensão 1 de $T Q \oplus T^{*} Q$ definido por $^{1}$

$$
\left(T Q \oplus T^{*} Q\right)_{L}=\left\{\left(q^{i}, \dot{q}^{i}, p_{i}\right) \mid p_{i} \dot{q}^{i}=0\right\},
$$

assim como as projeções

$$
\begin{aligned}
& \overrightarrow{\mathrm{pr}}: \quad T^{*}(T Q) \quad \longrightarrow \quad \vec{T}^{2 *} Q \\
& \left(q^{i}, \dot{q}^{i}, p_{i}, r_{i}\right) \longmapsto\left(q^{i}, \dot{q}^{i}, r_{i}\right){ }^{\prime}
\end{aligned}
$$

$\mathrm{e}$

$$
\begin{aligned}
& \text { pr : } \quad T^{*}(T Q) \quad \longrightarrow \quad T^{2 *} Q \\
& \left(q^{i}, \dot{q}^{i}, p_{i}, r_{i}\right) \longmapsto\left(q^{i}, \dot{q}^{i}, r_{i}, p_{i} \dot{q}^{i}\right){ }^{\prime}
\end{aligned}
$$

onde usamos que a coordenada $r$ possui a mesma lei de transformação (veja a equação (3.27)) que a expressão $p_{i} \dot{q}^{i}$ (veja as equações (3.22) e (3.30)). Tais mapas formam as seguintes sequências exatas de fibrados vetoriais sobre $T Q$ :

$$
0 \longrightarrow T Q \oplus T^{*} Q \stackrel{\overrightarrow{\mathrm{i}}}{\longrightarrow} T^{*}(T Q) \stackrel{\overrightarrow{\mathrm{pr}}}{\longrightarrow} \vec{T}^{2 *} Q \longrightarrow 0
$$

$\mathrm{e}$

$$
0 \longrightarrow\left(T Q \oplus T^{*} Q\right)_{L} \stackrel{\mathrm{i}}{\longrightarrow} T^{*}(T Q) \stackrel{\mathrm{pr}}{\longrightarrow} T^{2 *} Q \longrightarrow 0
$$

Considerando a forma canônica $\theta_{T Q}$ sobre $T^{\star}(T Q)$, que em coordenadas canônicas $\left(q^{i}, \dot{q}^{i}, p_{i}, r_{i}\right)$ é escrita como

$$
\theta_{T Q}=p_{i} d q^{i}+r_{i} d \dot{q}^{i},
$$

podemos compará-la com a correspondente forma de primeira ordem, a forma canônica $\theta_{Q}$ sobre $T^{*} Q$, que em coordenadas canônicas $\left(q^{i}, p_{i}\right)$ é escrita como

$$
\theta_{Q}=p_{i} d q^{i}
$$

Para tanto, é conveniente estender $\theta_{Q}$ de maneira natural a uma 1-forma $\theta_{Q}^{\prime}$ sobre $T Q \oplus T^{*} Q$, que em coordenadas canônicas $\left(q^{i}, \dot{q}^{i}, p_{i}\right)$ tem a mesma forma,

$$
\theta_{Q}^{\prime}=p_{i} d q^{i}
$$

Então vemos que o pull-back de $\theta_{T Q}$ por $\overrightarrow{\mathrm{i}}$ é igual a $\theta_{Q}^{\prime}$ e o pull-back de $\theta_{T Q}$ por i é igual à restrição de $\theta_{Q}^{\prime}$ à subvariedade $\left(T Q \oplus T^{*} Q\right)_{L}$ de $T Q \oplus T^{*} Q$. Por outro lado, quanto ao comportamento sob as projeções $\overrightarrow{\mathrm{pr}}$ e pr, podemos nos perguntar: a forma $\theta_{T Q}$ admite

\footnotetext{
${ }^{1} \mathrm{O}$ índice $L$ significa "Legendre", tendo em vista que a expressão $p_{i} \dot{q}^{i}$ ocupa uma posição central na teoria da transformação de Legendre.
} 
algum push-forward sob $\overrightarrow{p r}$ ou sob pr? Ou seja, existe alguma 1 -forma sobre $\vec{T}^{2 *} Q$ cujo pull-

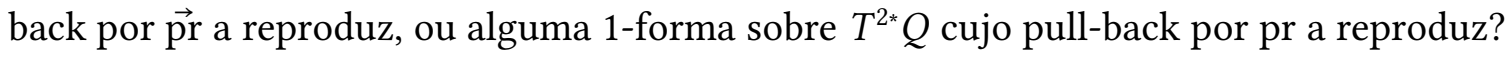
Como pr e pr são projeções de fibrados vetoriais, existe um critério para que isso seja o caso: considerando o campo de Euler do fibrado vetorial pertinente, é necessário e suficiente que a derivada de Lie de $\theta_{T Q}$ ao longo deste campo de Euler seja zero (veja, por exemplo, a Proposição A.1 do Apêndice da Ref. [6]). Agora, quanto à projeção pr, isso é fácil de decidir, pois nas coordenadas canônicas $\left(q^{i}, \dot{q}^{i}, p_{i}, r_{i}\right)$ de $T^{*}(T Q)$ e $\left(q^{i}, \dot{q}^{i}, r_{i}\right)$ de $\vec{T}^{2 *} Q$, o campo de Euler $\Sigma$ referente à projeção $\overrightarrow{\mathrm{pr}}$ é igual a

$$
\Sigma=p_{i} \frac{\partial}{\partial p_{i}}
$$

Assim, a derivada de Lie de $\theta_{T Q}$ ao longo de $\Sigma$ é

$$
L_{\Sigma} \theta_{T Q}=p_{i} d q^{i}
$$

que não é 0 . No caso da projeção pr, o argumento é um pouco mais complexo, pois o campo vetorial $\Sigma$ anterior serve como campo de Euler para a projeção pr apenas sobre a variedade $Q$, vista como subvariedade de $T Q$ através da seção zero, i.e., quando $\dot{q}=0$. Para lidar com a parte sobre $T Q \backslash\{0\}$, i.e., quando $\dot{q} \neq 0$, escolha qualquer métrica riemanniana $h$ sobre $Q$ e considere a lagrangiana da partícula livre se movendo sobre $Q$ com energia cinética dada por $h, L=\frac{1}{2} h_{k l} \dot{q}^{k} \dot{q}^{l}$, assim como a correspondente transformação de Legendre, dada por $p_{k}^{(0)}=\partial L / \partial \dot{q}^{k}=h_{k l} \dot{q}^{l}$; então temos $p_{k}^{(0)} \dot{q}^{k}=h_{k l} \dot{q}^{k} \dot{q}^{l}=2 L$ e portanto obtemos um campo de Euler $\Sigma_{L}$ referente à projeção pr como

$$
\Sigma_{L}=\left(p_{i}-\frac{p_{k} \dot{q}^{k}}{2 L} h_{i j} \dot{q}^{j}\right) \frac{\partial}{\partial p_{i}},
$$

Assim, a derivada de Lie de $\theta_{T Q}$ ao longo de $\Sigma_{L}$ é

$$
L_{\Sigma} \theta_{T Q}=\left(p_{i}-\frac{p_{k} \dot{q}^{k}}{2 L} h_{i j} \dot{q}^{j}\right) d q^{i}
$$

que também não é 0 . Concluímos então que a resposta à pergunta formulada acima é negativa: não existe nenhuma 1-forma sobre $\vec{T}^{2 *} Q$ cujo pull-back por $\overrightarrow{\mathrm{pr}}$ reproduz $\theta_{T Q}$, e nenhuma 1-forma sobre $T^{2 *} Q$ cujo pull-back por pr reproduz $\theta_{T Q}$.

Interpretamos esta impossibilidade de transportar a forma canõnica $\theta_{T Q}$, por pushforward, para o dual afim ou o dual linear do fibrado tangente de segunda ordem $T^{2} Q$ como um problema estrutural, pois configura um grave obstáculo contra tentativas de estender o formalismo simplético além de sistemas dinâmicos lagrangianos ou hamiltonianos de primeira ordem: já em segunda ordem, parece não existir nenhuma forma diferencial natural que poderia assumir o papel da forma canônica, ou ainda da forma simplética.

\subsubsection{Caso Multidimensional}

Para estudarmos o caso de interesse em teoria de campos, é necessário introduzir a estrutura de jatos de segunda ordem como foi apresentada na seção 1.4. Recapitulando brevemente o que foi visto naquela seção, o fibrado de jatos semi-holônomos de segunda 
ordem $\bar{J}^{2} E$ é um subfibrado de $J(J E)$, assim como o fibrado holônomo $J^{2} E$ que acomoda as segundas derivadas de seções da projeção $\pi: E \rightarrow M$. Qualquer sistema de coordenadas locais $\left(x^{\mu}, q^{i}\right)$ induz coordenadas locais $\left(x^{\mu}, q^{i}, q_{\mu}^{i}\right)$ de $J E,\left(x^{\mu}, q^{i}, q_{\mu}^{i}, q_{; \mu}^{i}, q_{\mu ; \nu}^{i}\right)$ de $J(J E)$, $\left(x^{\mu}, q^{i}, q_{\mu}^{i}, q_{\mu ; \nu}^{i}\right)$ de $\bar{J}^{2} E$ e $\left(x^{\mu}, q^{i}, q_{\mu}^{i}, q_{\mu \nu}^{i}\right)$ de $J^{2} E$. Se passarmos para um novo sistema de coordenadas locais $\left(x^{\prime v}, q^{\prime j}\right)$ de $E$, teremos a seguinte lei de transformação para as coordenadas induzidas de $J(J E)$,

$$
\begin{aligned}
q_{; \nu}^{\prime j}= & \frac{\partial q^{\prime j}}{\partial q^{i}} \frac{\partial x^{\mu}}{\partial x^{\prime v}} q_{; \mu}^{i}+\frac{\partial q^{\prime j}}{\partial x^{\mu}} \frac{\partial x^{\mu}}{\partial x^{\prime v}} \\
\vec{q}_{\alpha ; \beta}^{j}= & \frac{\partial q^{\prime j}}{\partial q^{i}} \frac{\partial x^{\mu}}{\partial x^{\prime \alpha}} \frac{\partial x^{v}}{\partial x^{\prime \beta}} \vec{q}_{\mu ; \nu}^{i}, \\
q_{\alpha ; \beta}^{\prime j}= & \frac{\partial q^{\prime j}}{\partial q^{i}} \frac{\partial x^{\mu}}{\partial x^{\prime \alpha}} \frac{\partial x^{v}}{\partial x^{\prime \beta}} q_{\mu ; \nu}^{i}+\frac{\partial^{2} q^{\prime j}}{\partial q^{i} \partial q^{k}} \frac{\partial x^{\mu}}{\partial x^{\prime \alpha}} \frac{\partial x^{v}}{\partial x^{\prime \beta}} q_{\mu}^{i} q_{; v}^{k} \\
& +\frac{\partial^{2} q^{\prime j}}{\partial q^{k} \partial x^{\mu}} \frac{\partial x^{\mu}}{\partial x^{\prime \alpha}} \frac{\partial x^{v}}{\partial x^{\prime \beta}} q_{; \nu}^{k}+\left(\frac{\partial^{2} q^{\prime j}}{\partial q^{i} \partial x^{v}} \frac{\partial x^{\mu}}{\partial x^{\prime \alpha}} \frac{\partial x^{v}}{\partial x^{\prime \beta}}-\frac{\partial^{2} x^{\prime \gamma}}{\partial x^{\kappa} \partial x^{\lambda}} \frac{\partial q^{\prime j}}{\partial q^{i}} \frac{\partial x^{\mu}}{\partial x^{\prime \gamma}} \frac{\partial x^{\kappa}}{\partial x^{\prime \alpha}} \frac{\partial x^{\lambda}}{\partial x^{\prime \beta}}\right) q_{\mu}^{i} \\
& +\frac{\partial^{2} q^{\prime j}}{\partial x^{\mu} \partial x^{v}} \frac{\partial x^{\mu}}{\partial x^{\prime \alpha}} \frac{\partial x^{v}}{\partial x^{\prime \beta}}-\frac{\partial^{2} x^{\prime \gamma}}{\partial x^{\kappa} \partial x^{\lambda}} \frac{\partial q^{\prime j}}{\partial x^{\mu}} \frac{\partial x^{\mu}}{\partial x^{\prime \gamma}} \frac{\partial x^{\kappa}}{\partial x^{\prime \alpha}} \frac{\partial x^{\lambda}}{\partial x^{\prime \beta}},
\end{aligned}
$$

a seguinte lei de transformação para as coordenadas induzidas de $\bar{J}^{2} E$,

$$
\begin{aligned}
q_{v}^{\prime j}= & \frac{\partial q^{\prime j}}{\partial q^{i}} \frac{\partial x^{\mu}}{\partial x^{\prime \nu}} q_{\mu}^{i}+\frac{\partial q^{j}}{\partial x^{\mu}} \frac{\partial x^{\mu}}{\partial x^{\prime v}} \\
\vec{q}_{\alpha ; \beta}^{\prime j}= & \frac{\partial q^{\prime j}}{\partial q^{i}} \frac{\partial x^{\mu}}{\partial x^{\prime \alpha}} \frac{\partial x^{v}}{\partial x^{\prime \beta}} \vec{q}_{\mu, v}^{i}, \\
q_{\alpha ; \beta}^{\prime j}= & \frac{\partial q^{\prime j}}{\partial q^{i}} \frac{\partial x^{\mu}}{\partial x^{\prime \alpha}} \frac{\partial x^{v}}{\partial x^{\prime \beta}} q_{\mu ; v}^{i}+\frac{\partial^{2} q^{\prime j}}{\partial q^{i} \partial q^{k}} \frac{\partial x^{\mu}}{\partial x^{\prime \alpha}} \frac{\partial x^{v}}{\partial x^{\prime \beta}} q_{\mu}^{i} q_{v}^{k} \\
& +\left(\frac{\partial^{2} q^{\prime j}}{\partial q^{i} \partial x^{v}} \frac{\partial x^{v}}{\partial x^{\prime \alpha}} \frac{\partial x^{\mu}}{\partial x^{\prime \beta}}+\frac{\partial^{2} q^{\prime j}}{\partial q^{i} \partial x^{v}} \frac{\partial x^{\mu}}{\partial x^{\prime \alpha}} \frac{\partial x^{v}}{\partial x^{\prime \beta}}-\frac{\partial^{2} x^{\prime \gamma}}{\partial x^{k} \partial x^{\lambda}} \frac{\partial q^{\prime j}}{\partial q^{i}} \frac{\partial x^{\mu}}{\partial x^{\prime \gamma}} \frac{\partial x^{\kappa}}{\partial x^{\prime \alpha}} \frac{\partial x^{\lambda}}{\partial x^{\prime \beta}}\right) q_{\mu}^{i} \\
& +\frac{\partial^{2} q^{\prime j}}{\partial x^{\mu} \partial x^{v}} \frac{\partial x^{\mu}}{\partial x^{\prime \alpha}} \frac{\partial x^{v}}{\partial x^{\prime \beta}}-\frac{\partial^{2} x^{\prime \gamma}}{\partial x^{\kappa} \partial x^{\lambda}} \frac{\partial q^{\prime j}}{\partial x^{\mu}} \frac{\partial x^{\mu}}{\partial x^{\prime \gamma}} \frac{\partial x^{\kappa}}{\partial x^{\prime \alpha}} \frac{\partial x^{\lambda}}{\partial x^{\prime \beta}},
\end{aligned}
$$

e a seguinte lei de transformação para as coordenadas induzidas de $J^{2} E$,

$$
\begin{aligned}
q_{v}^{\prime j}= & \frac{\partial q^{\prime j}}{\partial q^{i}} \frac{\partial x^{\mu}}{\partial x^{\prime \nu}} q_{\mu}^{i}+\frac{\partial q^{\prime j}}{\partial x^{\mu}} \frac{\partial x^{\mu}}{\partial x^{\prime v}} \\
\vec{q}_{\alpha \beta}^{\prime j}= & \frac{\partial q^{\prime j}}{\partial q^{i}} \frac{\partial x^{\mu}}{\partial x^{\prime \alpha}} \frac{\partial x^{v}}{\partial x^{\prime \beta}} \vec{q}_{\mu v}^{i}, \\
q_{\alpha \beta}^{\prime j}= & \frac{\partial q^{\prime j}}{\partial q^{i}} \frac{\partial x^{\mu}}{\partial x^{\prime \alpha}} \frac{\partial x^{v}}{\partial x^{\prime \beta}} q_{\mu v}^{i}+\frac{\partial^{2} q^{\prime j}}{\partial q^{i} \partial q^{k}} \frac{\partial x^{\mu}}{\partial x^{\prime \alpha}} \frac{\partial x^{v}}{\partial x^{\prime \beta}} q_{\mu}^{i} q_{v}^{k} \\
& +\left(\frac{\partial^{2} q^{\prime j}}{\partial q^{i} \partial x^{v}} \frac{\partial x^{v}}{\partial x^{\prime \alpha}} \frac{\partial x^{\mu}}{\partial x^{\prime \beta}}+\frac{\partial^{2} q^{\prime j}}{\partial q^{i} \partial x^{v}} \frac{\partial x^{\mu}}{\partial x^{\prime \alpha}} \frac{\partial x^{v}}{\partial x^{\prime \beta}}-\frac{\partial^{2} x^{\prime \gamma}}{\partial x^{k} \partial x^{\lambda}} \frac{\partial q^{j}}{\partial q^{i}} \frac{\partial x^{\mu}}{\partial x^{\prime \gamma}} \frac{\partial x^{\kappa}}{\partial x^{\prime \alpha}} \frac{\partial x^{\lambda}}{\partial x^{\prime \beta}}\right) q_{\mu}^{i} \\
& +\frac{\partial^{2} q^{\prime j}}{\partial x^{\mu} \partial x^{v}} \frac{\partial x^{\mu}}{\partial x^{\prime \alpha}} \frac{\partial x^{v}}{\partial x^{\prime \beta}}-\frac{\partial^{2} x^{\prime \gamma}}{\partial x^{k} \partial x^{\lambda}} \frac{\partial q^{\prime j}}{\partial x^{\mu}} \frac{\partial x^{\mu}}{\partial x^{\prime \gamma}} \frac{\partial x^{\kappa}}{\partial x^{\prime \alpha}} \frac{\partial x^{\lambda}}{\partial x^{\prime \beta}}
\end{aligned}
$$


Passando a duais, coordenadas locais $\left(x^{\mu}, q^{i}\right)$ de $E$ induzem coordenadas locais adaptadas $\left(x^{\mu}, q^{i}, q_{\mu}^{i}, p_{i}^{\mu \nu}, r\right) \operatorname{de} J^{2 \star} E,\left(x^{\mu}, q^{i}, q_{\mu}^{i}, p_{i}^{\mu \nu}\right)$ de $\vec{J}^{2 \star} E,\left(x^{\mu}, q^{i}, q_{\mu}^{i}, p_{i}^{\mu ; v}, \bar{r}\right) \operatorname{de} \bar{J}^{2 \star} E$ e $\left(x^{\mu}, q^{i}, q_{\mu}^{i}, p_{i}^{\mu ; \nu}\right)$ de $\overrightarrow{\bar{J}}^{2 *} E$. Tomando mais uma vez um novo sistema de coordenadas locais $\left(x^{\prime \nu}, q^{\prime j}\right)$ de $E$, teremos a seguinte lei de transformação para as coordenadas induzidas de $\bar{J}^{2 \star} E$ e $\vec{J}^{2 \star} E$,

$$
\begin{aligned}
p_{j}^{\prime \alpha ; \beta}= & \frac{\partial q^{i}}{\partial q^{\prime j}} \frac{\partial x^{\prime \alpha}}{\partial x^{\mu}} \frac{\partial x^{\prime \beta}}{\partial x^{v}} p_{i}^{\mu ; \nu} \\
\bar{r}^{\prime}= & \bar{r}-\left(\frac{\partial^{2} q^{\prime j}}{\partial q^{k} \partial q^{l}} \frac{\partial q^{i}}{\partial q^{\prime j}} q_{\mu}^{k} q_{v}^{l}+\frac{\partial^{2} q^{\prime j}}{\partial q^{k} \partial x^{\mu}} \frac{\partial q^{i}}{\partial q^{\prime j}} q_{v}^{k}+\frac{\partial^{2} q^{\prime j}}{\partial q^{k} \partial x^{v}} \frac{\partial q^{i}}{\partial q^{\prime j}} q_{\mu}^{k}-\frac{\partial^{2} x^{\prime \gamma}}{\partial x^{\mu} \partial x^{v}} \frac{\partial x^{\rho}}{\partial x^{\prime \gamma}} q_{\rho}^{i}\right) p_{i}^{\mu ; v} \\
& -\left(\frac{\partial^{2} q^{\prime j}}{\partial x^{\mu} \partial x^{v}} \frac{\partial q^{i}}{\partial q^{\prime j}}-\frac{\partial^{2} x^{\prime \gamma}}{\partial x^{\mu} \partial x^{v}} \frac{\partial x^{\rho}}{\partial x^{\prime \gamma}} \frac{\partial q^{i}}{\partial q^{\prime j}} \frac{\partial q^{\prime j}}{\partial x^{\rho}}\right) p_{i}^{\mu ; v}
\end{aligned}
$$

e a seguinte lei de transformação para as coordenadas induzidas de $J^{2 \star} E$ e $\vec{J}^{2 \star} E$,

$$
\begin{aligned}
p_{j}^{\prime \alpha \beta}= & \frac{\partial q^{i}}{\partial q^{\prime j}} \frac{\partial x^{\prime \alpha}}{\partial x^{\mu}} \frac{\partial x^{\prime \beta}}{\partial x^{v}} p_{i}^{\mu v} \\
r^{\prime}= & r-\left(\frac{\partial^{2} q^{\prime j}}{\partial q^{k} \partial q^{l}} \frac{\partial q^{i}}{\partial q^{\prime j}} q_{\mu}^{k} q_{v}^{l}+\frac{\partial^{2} q^{\prime j}}{\partial q^{k} \partial x^{\mu}} \frac{\partial q^{i}}{\partial q^{\prime j}} q_{v}^{k}+\frac{\partial^{2} q^{j}}{\partial q^{k} \partial x^{v}} \frac{\partial q^{i}}{\partial q^{\prime j}} q_{\mu}^{k}-\frac{\partial^{2} x^{\prime \gamma}}{\partial x^{\mu} \partial x^{v}} \frac{\partial x^{\rho}}{\partial x^{\prime \gamma}} q_{\rho}^{i}\right) p_{i}^{\mu v} \\
& -\left(\frac{\partial^{2} q^{\prime j}}{\partial x^{\mu} \partial x^{v}} \frac{\partial q^{i}}{\partial q^{\prime j}}-\frac{\partial^{2} x^{\prime \gamma}}{\partial x^{\mu} \partial x^{v}} \frac{\partial q^{i}}{\partial q^{\prime j}} \frac{\partial q^{\prime j}}{\partial x^{\rho}} \frac{\partial x^{\rho}}{\partial x^{\prime \gamma}}\right) p_{i}^{\mu \nu}
\end{aligned}
$$

Nota-se que esses espaços de cojatos não possuem estrutura multissimplética óbvia. Sendo assim, nossa atenção se volta para o fibrado dual $J^{\star}(J E)$ do fibrado de jatos iterado $J(J E)$, de coordenadas induzidas $\left(x^{\mu}, q^{i}, q_{\mu}^{i}, p_{i}^{\mu}, p_{i}^{\mu ; \nu}, p\right)$. Os elementos de $J^{\star}(J E)$ são definidos através do pareamento com os elementos de $J(J E)$, que em termos das coordenadas induzidas pertinentes assume a forma

$$
p_{i}^{\mu ; v} q_{\mu ; \nu}^{i}+p_{i}^{\mu} q_{; \mu}^{i}+p
$$

Então, se passarmos para um novo sistema de coordenadas locais $\left(x^{\prime v}, q^{\prime j}\right)$, devemos ter

$$
p_{i}^{\mu ; v} q_{\mu ; v}^{i}+p_{i}^{\mu} q_{; \mu}^{i}+p=p_{j}^{\prime \alpha ; \beta} q_{\alpha ; \beta}^{\prime j}+p_{j}^{\prime v} q_{; v}^{j}+p^{\prime},
$$

e portanto a lei de transformação para as coordenadas induzidas de $J^{\star}(J E)$ será

$$
\begin{aligned}
p_{j}^{\prime \alpha ; \beta}= & \frac{\partial q^{i}}{\partial q^{\prime j}} \frac{\partial x^{\prime \alpha}}{\partial x^{\mu}} \frac{\partial x^{\prime \beta}}{\partial x^{v}} p_{i}^{\mu ; \nu}, \\
p_{j}^{\prime \nu}= & \frac{\partial q^{i}}{\partial q^{\prime j}} \frac{\partial x^{\prime \nu}}{\partial x^{\mu}}\left(p_{i}^{\mu}-\frac{\partial^{2} q^{\prime m}}{\partial q^{i} \partial q^{k}} \frac{\partial q^{l}}{\partial q^{\prime m}} q_{\kappa}^{k} p_{l}^{\kappa ; \mu}-\frac{\partial^{2} q^{\prime m}}{\partial q^{i} \partial x^{\kappa}} \frac{\partial q^{l}}{\partial q^{\prime m}} p_{l}^{\kappa ; \mu}\right) \\
p^{\prime}= & p-\frac{\partial q^{i}}{\partial q^{\prime j}} \frac{\partial q^{\prime j}}{\partial x^{\mu}} p_{i}^{\mu} \\
& +\frac{\partial^{2} q^{\prime m}}{\partial q^{i} \partial q^{k}} \frac{\partial q^{l}}{\partial q^{\prime m}} \frac{\partial q^{i}}{\partial q^{\prime j}} \frac{\partial q^{\prime j}}{\partial x^{\mu}} q_{\kappa}^{k} p_{l}^{\kappa ; \mu}-\frac{\partial^{2} q^{\prime m}}{\partial q^{i} \partial x^{\kappa}} \frac{\partial q^{l}}{\partial q^{\prime m}} q_{\mu}^{i} p_{l}^{\mu ; \kappa}+\frac{\partial^{2} x^{\prime \gamma}}{\partial x^{\mu} \partial x^{v}} \frac{\partial x^{\rho}}{\partial x^{\prime \gamma}} q_{\rho}^{i} p_{i}^{\mu ; \nu} \\
& -\frac{\partial^{2} q^{\prime m}}{\partial x^{\mu} \partial x^{v}} \frac{\partial q^{l}}{\partial q^{\prime m}} p_{l}^{\mu ; \nu}+\frac{\partial^{2} x^{\prime \gamma}}{\partial x^{\mu} \partial x^{v}} \frac{\partial x^{\rho}}{\partial x^{\prime \gamma}} \frac{\partial q^{l}}{\partial q^{\prime j}} \frac{\partial q^{\prime j}}{\partial x^{\rho}} p_{l}^{\mu ; \nu}+\frac{\partial^{2} q^{\prime m}}{\partial q^{i} \partial x^{\kappa}} \frac{\partial q^{l}}{\partial q^{\prime m}} \frac{\partial q^{i}}{\partial q^{\prime j}} \frac{\partial q^{\prime j}}{\partial x^{\mu}} p_{l}^{\kappa ; \mu}
\end{aligned}
$$


Todas essas leis de transformação para os diversos duais permanecem quase que inalteradas, exceto pelo surgimento de uma fator do tipo $\operatorname{det}\left(\partial x^{\prime} / \partial x\right)$ ou $\operatorname{det}\left(\partial x / \partial x^{\prime}\right)$, quando substituirmos o dual comum pelo dual torcido, definido como o produto tensorial do dual comum com o fibrado em linhas obtido como pull-back adequado do fibrado em linhas das formas de volume sobre a variedade base $M, \wedge^{n} T^{*} M$. Agora, segue destas leis de transformação que os seguintes mapas são bem definidos e são morfismos de fibrados vetoriais sobre $J E$ : as inclusões

$$
\begin{array}{cccc}
\overrightarrow{\mathrm{i}}: & \pi_{J E}^{*}\left(\vec{J}^{\oplus} E\right) & \longrightarrow & \vec{J}^{\oplus}(J E) \\
\left(x^{\mu}, q^{i}, q_{\mu}^{i}, p_{i}^{\mu}\right) & \longmapsto & \left(x^{\mu}, q^{i}, q_{\mu}^{i}, p_{i}^{\mu}, 0\right)
\end{array}
$$

$\mathrm{e}$

$$
\text { i : } \begin{array}{ccc}
\left(\pi_{J E}^{*}\left(J^{\circledast} E\right)\right)_{L} & \longrightarrow & J^{\oplus}(J E) \\
\left(x^{\mu}, q^{i}, q_{\mu}^{i}, p_{i}^{\mu}, p\right) & \longmapsto & \left(x^{\mu}, q^{i}, q_{\mu}^{i}, p_{i}^{\mu}, 0, p\right)
\end{array},
$$

onde $\left(\pi_{J E}^{*}\left(J^{\circledast} E\right)\right)_{L}$ é o subfibrado vetorial de codimensão 1 de $\pi_{J E}^{*}\left(J^{\circledast} E\right)$ definido por

$$
\left(\pi_{J E}^{\star}\left(J^{\circledast} E\right)\right)_{L}=\left\{\left(x^{\mu}, q^{i}, q_{\mu}^{i}, p_{i}^{\mu}, p\right) \mid p_{i}^{\mu} q_{\mu}^{i}+p=0\right\},
$$

assim como as projeções

$$
\begin{aligned}
& \overrightarrow{\mathrm{pr}}: \quad \vec{J}^{\oplus}(J E) \quad \longrightarrow \quad \overrightarrow{\bar{J}}^{2 \oplus} E \\
& \left(x^{\mu}, q^{i}, q_{\mu}^{i}, p_{i}^{\mu}, p_{i}^{\mu ; \nu}\right) \longmapsto\left(x^{\mu}, q^{i}, q_{\mu}^{i}, p_{i}^{\mu ; \nu}\right)
\end{aligned}
$$

$\mathrm{e}$

$$
\begin{array}{ccc}
\text { pr : } & J^{\circledast}(J E) & \bar{J}^{2 \circledast} E \\
\left(x^{\mu}, q^{i}, q_{\mu}^{i}, p_{i}^{\mu}, p_{i}^{\mu ; \nu}, p\right) & \longmapsto & \left(x^{\mu}, q^{i}, q_{\mu}^{i}, p_{i}^{\mu ; \nu}, p_{i}^{\mu} q_{\mu}^{i}+p\right)
\end{array},
$$

onde usamos que, mediante multiplicação pelo fator $\operatorname{det}\left(\partial x^{\prime} / \partial x\right)$, a coordenada $\bar{r}$ possui a mesma lei de transformação (veja a equação (3.51)) que a expressão $p_{i}^{\mu} q_{\mu}^{i}+p$ (veja as equações (3.44), (3.57) e (3.58)). Tais mapas formam as seguintes sequências exatas de fibrados vetoriais sobre $J E$ :

$$
0 \longrightarrow \pi_{J E}^{*}\left(\vec{J}^{\circledast} E\right) \stackrel{\overrightarrow{\mathrm{i}}}{\longrightarrow} \vec{J}^{\circledast}(J E) \stackrel{\overrightarrow{\mathrm{pr}}}{\longrightarrow} \overrightarrow{\bar{J}}^{2 \oplus} E \longrightarrow 0
$$

$\mathrm{e}$

$$
0 \longrightarrow\left(\pi_{J E}^{*}\left(J^{\circledast} E\right)\right)_{L} \stackrel{\mathrm{i}}{\longrightarrow} J^{\circledast}(J E) \stackrel{\mathrm{pr}}{\longrightarrow} \bar{J}^{2 \circledast} E \longrightarrow 0
$$

Considerando a forma policanônica $\hat{\theta}_{J E}$ sobre $\vec{J}^{\oplus}(J E)$, que em coordenadas canônicas $\left(x^{\mu}, q^{i}, q_{\mu}^{i}, p_{i}^{\mu}, p_{i}^{\mu ; \nu}\right)$ é escrita como

$$
\hat{\theta}_{J E}=p_{i}^{\mu} d q^{i} \otimes d^{n} x_{\mu}+p_{i}^{\mu ; v} d q_{v}^{i} \otimes d^{n} x_{\mu}
$$

e a forma multicanônica $\theta_{J E}$ sobre $J^{\oplus}(J E)$, que em coordenadas canônicas $\left(x^{\mu}, q^{i}, q_{\mu}^{i}, p_{i}^{\mu}, p_{i}^{\mu ; \nu}, p\right)$ é escrita como

$$
\theta_{J E}=p_{i}^{\mu} d q^{i} \wedge d^{n} x_{\mu}+p_{i}^{\mu ; v} d q_{v}^{i} \wedge d^{n} x_{\mu}+p d^{n} x
$$

podemos compará-las com as respectivas formas canônicas de primeira ordem, a poli- 
simplética sobre $\vec{J}^{\circledast} E$ e a multissimplética sobre $J^{\circledast} E$, que em coordenadas canônicas são escritas como

$$
\begin{gathered}
\hat{\theta}_{E}=p_{i}^{\mu} d q^{i} \otimes d^{n} x_{\mu} \\
\theta_{E}=p_{i}^{\mu} d q^{i} \wedge d^{n} x_{\mu}+p d^{n} x .
\end{gathered}
$$

Para tanto, é necessário estender estas de maneira natural a formas $\hat{\theta}_{E}^{\prime}$ sobre $\pi_{J E}^{*}\left(\vec{J}^{\circledast} E\right)$ e $\theta_{E}^{\prime}$ sobre $\pi_{J E}^{\star}\left(J^{\circledast} E\right)$, que em coordenadas canônicas tem a mesma forma,

$$
\begin{gathered}
\hat{\theta}_{E}^{\prime}=p_{i}^{\mu} d q^{i} \otimes d^{n} x_{\mu} \\
\theta_{E}^{\prime}=p_{i}^{\mu} d q^{i} \wedge d^{n} x_{\mu}+p d^{n} x .
\end{gathered}
$$

Então vemos que o pull-back de $\hat{\theta}_{J E}$ por $\overrightarrow{\mathrm{i}}$ é igual a $\hat{\theta}_{E}^{\prime}$ e o pull-back de $\theta_{J E}$ por i é igual à restrição de $\theta_{E}^{\prime}$ à subvariedade $\left(\pi_{J E}^{*}\left(J^{\circledast} E\right)\right)_{L}$ de $\pi_{J E}^{*}\left(J^{\circledast} E\right)$. Por outro lado, quanto ao comportamento sob as projeções $\overrightarrow{\mathrm{pr}}$ e pr, podemos fazer a mesma pergunta que antes: as formas $\hat{\theta}_{J E}$ e $\theta_{J E}$ admitem algum push-forward sob $\overrightarrow{\mathrm{pr}}$ e sob pr, respectivamente? Ou seja, existe alguma forma sobre $\overrightarrow{\bar{J}}^{2 \oplus} E$ cujo pull-back por $\overrightarrow{\text { pr }}$ reproduz $\hat{\theta}_{J E}$, ou alguma forma sobre $\bar{J}^{2 \circledast} E$ cujo pull-back por pr reproduz $\theta_{J E}$ ?

Novamente, $\overrightarrow{p r}$ e pr são projeções de fibrados vetoriais, e portanto podemos tentar usar o mesmo critério que antes. Para isso, é muito útil perceber que o critério apresentado na Proposição A.1 do Apêndice da Ref. [6], segundo o qual uma forma diferencial $\alpha$ sobre o espaço total de um fibrado vetorial admite um push-forward para uma forma diferencial sobre a variedade base se e somente se sua derivada de Lie ao longo do campo de Euler se anula, continua valendo da mesma forma se substituirmos o campo de Euler por qualquer outro campo vetorial vertical sobre o espaço total cujo fluxo converge para a seção zero quando $t \rightarrow-\infty$. No caso polissimplético, não precisamos desta generalização, pois o

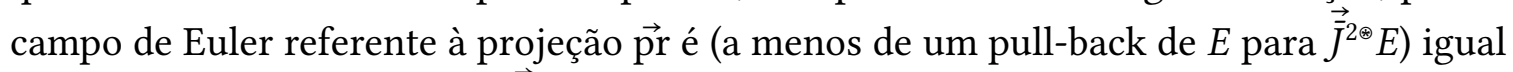
ao campo de Euler padrão de $\vec{J}^{\circledast} E$ como fibrado vetorial sobre $E$,

$$
\Sigma=p_{i}^{\mu} \frac{\partial}{\partial p_{i}^{\mu}}
$$

Além disso, é fácil calcular a derivada de Lie de $\hat{\theta}_{J E}$ ao longo de $\Sigma$,

$$
L_{\Sigma} \hat{\theta}_{J E}=\hat{\theta}_{E}^{\prime}
$$

que não é 0 . Mas no caso multissimplético, a generalização torna-se útil, pois o campo de Euler referente à projeção pr é (a menos de um pull-back de $E$ para $\bar{J}^{2 \circledast} E$ ) igual a

$$
\Sigma^{\prime}=p_{i}^{\mu} \frac{\partial}{\partial p_{i}^{\mu}}-p_{i}^{\mu} q_{\mu}^{i} \frac{\partial}{\partial p}
$$

que difere ligeiramente do campo de Euler padrão de $J^{\circledast} E$ como fibrado vetorial sobre $E$, 


$$
\Sigma=p_{i}^{\mu} \frac{\partial}{\partial p_{i}^{\mu}}+p \frac{\partial}{\partial p} .
$$

Novamente, é fácil calcular a derivada de Lie de $\theta_{J E}$ ao longo de $\Sigma^{\prime}$,

$$
L_{\Sigma^{\prime}} \theta_{J E}=p_{i}^{\mu} d q^{i} \wedge d^{n} x_{\mu}-p_{i}^{\mu} q_{\mu}^{i} d^{n} x
$$

que não é 0 . Concluímos então que, mais uma vez, a resposta à pergunta formulada acima é negativa: não existe nenhuma forma sobre ${\overrightarrow{J^{2}}}^{\circledR} E$ cujo pull-back por $\overrightarrow{\text { pr reproduz }} \hat{\theta}_{J E}$, e nenhuma forma sobre $\bar{J}^{2 \circledast} E$ cujo pull-back por pr reproduz $\theta_{J E}$.

Nenhuma dessas conclusões se altera se substituirmos os duais dos fibrados de jatos semi-holônomos pelos duais dos fibrados de jatos holônomos, por simetrização.

Interpretamos esta impossibilidade de transportar as formas canônicas $\hat{\theta}_{J E}$ e $\theta_{J E}$, por push-forward, para o dual afim ou o dual linear do fibrado de jatos holônomos de segunda ordem $J^{2} E$ como um problema estrutural, pois configura um grave obstáculo contra tentativas de estender o formalismo simplético além de sistemas dinâmicos lagrangianos ou hamiltonianos de primeira ordem: já em segunda ordem, parece não existir nenhuma forma diferencial natural que poderia assumir o papel da forma (poli- ou multi-) canônica, ou ainda da forma (poli- ou multi-) simplética. 


\section{Referências Bibliográficas}

[1] A. R. Tarrida, Affine Maps, Euclidean Motions and Quadrics, Springer, Londom (2011), DOI: https://doi.org/10.1007/978-0-85729-710-5.

[2] M. Forger \& S.V. Romero, Covariant Poisson Brackets in Geometric Field Theory, Commun. Math. Phys. 256 375-410 (2005), DOI: https://doi.org/10.1007/ S00220-005-1287-8.

[3] M. Forger \& F. Antoneli, Fibrados, Conexões e Classes Características, IME-USP 2011, https://www.ime.usp.br/ forger/pdffiles/fibrados.pdf.

[4] R. Abraham \& J.E. Marsden, Foundations of Mechanics, $2^{\text {nd }}$ edition, Benjamin/ Cummings, London 1987.

[5] M. Berger, Geometry, Vol. I, Springer, Berlin 2009.

[6] M. Forger, C. Paufler \& H. Römer, Hamiltonian Multivector Fields and Poisson Forms in Multisymplectic Field Theory, J. Math. Phys. 46 (2005) 112903, 29 pp., DOI: https: //doi.org/10.1063/1.2116320.

[7] B.T. Costa, M. Forger \& L. H. P. Pêgas, Lie Groupoids in Classical Field Theory I: Noether's Theorem, J. Geom. Phys. 131 (2018) 220-245, DOI: https://doi.org/10.1016/j. geomphys.2018.03.015.

[8] M. Forger \& L.G. Gomes, Multisympletic and Polysympletic Structures on Fiber Bundles, Rev. Math. Phys. 25 (2013) 1350018, 47 pp., DOI: https://doi.org/10.1142/ S0129055X13500189.

[9] D. J. Saunders, The geometry of jet bundles, Cambridge University Press, Cambridge 1989.

[10] J. M. Masqué, Poincaré Cartan Forms in Higher Order Variational Calculus on Fibred Manifolds, Rev. Matemática Iberoamericana 4 (1985) 85-126, http://eudml.org/doc/ 39833.

[11] W. F. Shadwick, The Hamiltoinian Formulation of Regular rth-order Lagrangian Field Theories, Lett. Math. Phys. 6 (1982) 409-416, DOI: https://doi.org/10.1007/BF00405859.

[12] Ivan Kolár, A Geometrical Version of the Higher Order Hamilton Formalism in Fibred Manifolds, J. Geom. Phys. 2 (1984) 127-137, DOI: https://doi.org/10.1016/ 0393-0440(84)90007-X. 